\title{
Angiographic Findings in Cases with a History of Severe Retinopathy of Prematurity Treated with anti-VEGFs Follow-up to Age 6 Years
}

Hande Celiker ( $\nabla$ drhandeceliker@yahoo.com )

Marmara University School of Medicine https://orcid.org/0000-0002-1683-0725

Ozlem Sahin

Marmara University School of Medicine Head of Ophthalmology Department

\section{Research Article}

Keywords: anti-VEGFs, fluorescein angiography, late recurrence, RetCam, retinopathy of prematurity

Posted Date: June 17th, 2021

DOI: https://doi.org/10.21203/rs.3.rs-335877/v1

License: (c) (i) This work is licensed under a Creative Commons Attribution 4.0 International License. Read Full License

Version of Record: A version of this preprint was published at International Ophthalmology on November 2 nd, 2021. See the published version at https://doi.org/10.1007/s10792-021-02119-y. 


\section{Abstract}

Purpose: To report the effects of anti-vascular endothelial growth factor (VEGF) treatment in vascular development for cases of acute retinopathy of prematurity (ROP) using fluorescent angiography (FA) and to present the results of our observational approach to retinal sequelae.

Methods: A total of 31 eyes in 19 patients with a history of treatment with anti-VEGF agents for classic type 1 ROP and aggressive posterior ROP who underwent FA between March 2014 to February 2020 were reviewed. Angiograms of retinal developmental features of patients aged 4 months to 6 years were examined.

Results: The patients mean gestational age were $26.06 \pm 1.90$ weeks and the mean birth weight were $837.68 \pm 236.79 \mathrm{~g}$. All cases showed various abnormalities at the vascular and avascular retina, and the posterior pole. All but one case showed a peripheral avascular area on FA evaluation during the follow-up period. We did not apply prophylactic laser treatment to these avascular retina. On the final examination, except one case, we did not observe any late reactivation in any patients.

Conclusion: FA is an important tool for assessing vascular maturation in infants. Every leakage should not be assumed to be evidence of late activation, as some leaks may be related to vascular immaturity. Retinal vascularization may not be completed in all patients, however this does not mean that all these patients need prophylactic laser application. Our observational approach may be more daring than the reports frequently encountered in the literature, but it should be noted that unnecessary laser treatment will also eliminate all the advantages of anti-VEGF treatment.

\section{Introduction}

Retinopathy of prematurity (ROP) is a proliferative retinopathy of premature infants that has been accepted as a major cause of irreversible visual deterioration and is present in both developed and developing countries [1-4]. Based on the results of two cardinal clinical trials (Cryotherapy for Retinopathy of Prematurity trial and the Early Treatment for Retinopathy of Prematurity trial), standard treatment options include cryotherapy or laser photocoagulation treatment (LPT) [5, 6]. Peripheral avascular retina is known for producing vascular endothelial growth factor (VEGF), thus complete ablation of avascular areas has been the gold standard therapy for the last 30 years. More recently, intravitreal anti-VEGF treatment has emerged as a new and more effective treatment approach for ROP [7]. The prospective randomized stratified multicenter clinical trial Bevacizumab Eliminates the Angiogenic Threat of Retinopathy of Prematurity Study (BEAT-ROP) assessed intravitreal injection of bevacizumab (IVB) monotherapy [7]. It reported that when considering both Zone 1 and posterior Zone 2 ROP together, IVB therapy was more effective than LPT [7]. Treatment with IVB, a humanized monoclonal antibody against VEGF-A, has obtained popularity after the publication of the BEAT-ROP study. Ranibizumab, an antibody fragment of humanized antiVEGF monoclonal antibody, is an alternative for intravitreal anti-VEGF therapy in ROP as well. Ranibizumab has a shorter half- life, higher binding affinity to VEGF than bevacizumab, and antibodies lacking a crystallizable fragment, thus, theoretically, may provoke fewer systemic adverse affects than treatment with bevacizumab [8].

Although these novel treatments show promise, there remain questions about intravitreal anti-VEGF treatments for ROP patients, such as the presence of avascular peripheral retina with chronic vascular arrest, the necessity of prophylactic LPT to the remnant avascular retinal areas, hallmarks of the late reactivation, and the optimum follow-up time of such cases. Due to the current lack of established guidelines on the handling of these patients, follow-up protocols and the extent to which prophylactic LPT is needed are still undefined. In this article, we aimed to present early and persistent effects of treatment with anti-VEGF agents on retinal maturation during the follow-up period utilizing FA results. Our secondary purpose was to discuss the long-term effects of our observational approach without any prophylactic ablative treatment on these peripheral zones of avascular retina.

\section{Methods}

\section{Study Description}

This is a single-center, retrospective, non-comperative case series study conducted at the Retinopathy of Prematurity Department at the Marmara University School of Medicine in Istanbul. The study protocol was approved by the local Institutional Ethics Board (The ethics committee approval number: 09.2016.654) and conducted according to the tenets of the Declaration of Helsinki. Informed consent was acquired from the parents for all examinations, including binocular indirect ophthalmoscopic (IO) examination with scleral depression, FA, and treatment, after detailed explanation of the different treatment options (LPT, IVB or intravitreal injection of ranibizumab (IVR)). All examinations (including FA screening) and treatments (intravitreal injections and LPT) were performed by an experienced ROP specialist (H.C.). Neonates with type I Zone I-II ROP and with aggressive posterior ROP (APROP) were enrolled in the present study. Type I ROP was defined as (1) Zone I with any stage and plus disease or (2) Zone I and Stage III without plus disease, or (3) Zone II Stage II or III with plus disease [6]. The diagnosis of plus disease $\left(^{+}\right)$could be made if sufficient venous dilatation and arteriolar tortuosity of the posterior retinal vessels were present in at least 2 quadrants of the eye and could later increase in severity to include iris vascular engorgement, poor pupillary dilatation, and vitreous haze. Cases with APROP were classified according to the revised International Classification of ROP [9]. APROP was defined as ROP with posterior location, prominence of plus disease, and ill-defined nature of the retinopathy. APROP generally does not progress through the typical stages of classic ROP disease and may emerge only as a flat neovascularization at the vascular and avascular junction of the retina [9]. For the purpose of defining the anteroposterior location of the retinal involvement has been described according to ICROP [9]. Zone I referring to a circle whose radius extends from the optic disk (OD) and whose length is twice the distance between the center of the OD and the center of the macula. Zone II resembles an annulus and encircles Zone I. It extends from Zone I to the nasal extent of the retina. Zone II posterior surrounds Zone I and has an external 
circumference based on a radius (originating at the center of the OD) whose length is three times the distance between the center of the disc and the center of the macula. Zone III is the remaining crescent of retina, primarily on the temporal side [7].

Examination, ROP Classification and Fluorescein Angiography (FA)

All fundus examinations were performed by IO, and digital RetCam retinal images were taken by RetCam ${ }^{\circledR}$ III (Clarity Medical Systems, Pleasanton, CA). If informed consent was given by parents or guardians, and the baby's systemic condition was appropriate, FA was also performed by RetCam ${ }^{\circledR}$ III with FA module. ROP was classified on the 10 of retina and the retinal images in accordance with classical ICROP criteria [10]. FA imaging was arranged before the treatment and then after the treatment within the first week; then subsequently, between 4- 6 weeks, between 12-14 weeks, at the sixth month, at the first year, every 3 months until the age of 2, every 6 months until the age of 4 , and then arranged annually, according to the late recurrence risks after treatment. Follow-up frequencies varied according to the physician's opinion. Infrequently, all scheduled angiograms could not be performed at the planned time due to irrelevant infantile complications or familial/technical problems. Under general anesthesia (GA), fundus images and FA of the posterior pole and peripheral retina were obtained using a neonatal lid speculum, scleral depressor, and RetCam ${ }^{\circledR}$ III. All babies were kept under rigorous observation and monitoring by their treating neonatologist and an experienced neonate anesthesiologist with detailed systemic evaluations. Neonates were appraised for any adverse events the day before and after the FA and treatment. The pupils were dilated using $0.5 \%$ phenylephrine and $0.5 \%$ tropicamide eye drops administered 3 times at 10-minute intervals. FA was performed using a bolus of sodium fluorescein administered intravenously at a dose of $0.1 \mathrm{~mL} / \mathrm{kg}$, followed by an isotonic saline flush. During the procedures, vital signs were tracked by a neonatologist (where necessary) and anesthesiologist. FA images were recorded in early, middle, and late phases. Images of the posterior pole, peripheral retina, and fibrovascular tissue were recorded for about 8 minutes until fluorescence had disappeared from the eye. We evaluated the angiographic characteristics and their variations, noting choroidal filling pattern and posterior pole changes (including the macula), features of the junction between vascular and avascular retina, and abnormalities in anterior and posterior within the vascular and avascular retina [11]. Extension of avascular retina more than 2 disc diameters (DD) from the ora serrata was considered to be a sign of peripheral nonperfusion [12].

\section{Anti-VEGF Administration}

The intravitreal injections were performed under GA in the operating theater under strict sterile conditions and using sterile equipment after meticulous flushing of the conjunctival cul-de-sac with $10 \%$ povidone-iodine (Batticon ${ }^{\circledR}$; Adeka, Istanbul, Turkey). After a standard asepsis procedure, an intravitreal injection of $0.625 \mathrm{mg} / 0.025 \mathrm{ml}$ of bevacizumab (Avastin ${ }^{\circledR}$; Genentech Inc., San Francisco, California, USA), or $0.25 \mathrm{mg} / 0.025 \mathrm{ml}$ of ranibizumab (Lucentis ${ }^{\circledR}$; Novartis, Basel, Switzerland) with a 30 -gauge needle $1.5 \mathrm{~mm}$ posterior to the limbus through the pars plicata in the superotemporal quadrants was performed. The tip of the needle was oriented perpendicular to the floor to lower the risk of crystalline lens damage. After the removal of the needle, simultaneous occlusion of the sclerotomy site using a sterile cotton-tipped applicator was performed. Topical netilmicin sulphate $0.3 \%$ was administered before the removal of the eye speculum. In bilateral cases, both eyes were injected on the same day using different equipment. After the procedure, the treated eyes were examined with 10 to check for any lens and/or retinal damage, vitreous hemorrhage, retinal detachment, optic nerve perfusion, or pulsations of the retinal artery. After surgery, potential systemic adverse effects of the anti-VEGF agents were monitored by their treating neonatologist. The babies received netilmicin sulphate $0.3 \%$ eye drop q6 hrs for 7 days after the injection. The postoperative examinations were implemented without anesthesia or sedation. The 10 examination and RetCam imaging were given on every visit. The infants were followed-up at 1, 3, 5, and 7 days postoperatively and then weekly for 2 months, followed by every 2 weeks until 52 weeks postmenstrual age (PMA), and then monthly until 1 year of age. The regression criteria of the disease were determined as follows: (1) presence of retinal vessels which progress to vascularization peripheral avascular retina, (2) regression of extraretinal fibrovascular proliferation (EFP), ridge, and demarcation line, (3) decrease in retinal vascular tortuosity and engorgement, disappearance of plus/preplus disease, (4) regression of neovascular proliferations. Existence of early recurrence was defined as lack of adequate regression after the treatment. Recurrence of the disease was noted as follows: (1) arrest of anterior progression of retinal vasculature associated with a new demarcation line, ridge, or EFP, (2) persistence or return of plus disease and/or neovascularizations, (3) angiographic reappearance of leakage.

\section{Results}

\section{Patients' General Characteristics and Reactivation of the Disease}

From March 2014 to February 2020, a total of 31 eyes in 19 patients ( 8 males and 11 females) were reviewed. The mean gestational age and mean birth weight of the patients was $26.06 \pm 1.90$ weeks (range, 23 to 31 weeks) and $837.68 \pm 236.79 \mathrm{~g}$ (range, 555 to $1580 \mathrm{~g}$ ), respectively. The mean age at the time of treatment was $36.61 \pm 2.85$ weeks (range, 32 to 42.3 weeks) PMA, and the mean follow-up period was $39.73 \pm 24.06$ months (range, 4 to 72 months). After all of the injections, no serious ocular or systemic complications were observed. Insignificant subconjunctival haemorrhage was noted in all of the infants treated with anti-VEGF. All of the eyes (100\%) demonstrated initial regression (especially in severity of venous tortuosity and arterial dilation) a few days after the injection. During the follow-up period, 3 eyes (9.7\%) of 3 cases (15.8\%) had reactivation and continued progression of ROP after a single IVB treatment. One of them (Case 8) experienced LPT at 41 weeks of PMA. The others (Case 5 and 17) underwent repeated IVB injections due to unregressed Stage $3^{+}$disease at $37^{\text {th }}$ weeks and $42^{\text {nd }}$ weeks of PMA, respectively. In Case 5 , laser treatment was suggested due to constant retinal elevation, but it was refused by the parents. During the follow-up period of Case 5, the remnant EFP at the vascular-avascular border regressed slightly, and vascularisation progressed on the temporal peripheral retina within the elevated fibrous tissue (Fig. 1). Although this ridge area never fully regressed, it did not elevate the retina. Nonetheless, at 104 weeks of PMA, the former EFP was observed as an elevated driblet that was dragging the retina. A FA exam revealed some 
leakage. Barrage laser treatment was applied posterior of this elevation, and subsequently, it partially regressed and dye leakage on FA was absent. None of the infants required surgery (either scleral buckling or 3-port pars plana vitrectomy). The general characterictics of patients are summarised in Table 1.

\section{FA Features}

Before and after the treatment, all the cases showed various angiographic changes during the early and late period of the disease. All the patients were monitored with FA according to our standard schedule. Vascular and structural abnormalities detected by FA were classified according to the definitions recommended by Lepore et al.[11] and taking into account our own observations. The general FA findings, vascular characteristics of the vascularavascular ( $\mathrm{V}$-Av) junction, features of the vascularised zone, posterior pole with macular abnormalities, and choroidal filling pattern are detailed in subsections of this paper. All the figures of the cases were arranged by age considering the changes in angiographic features according to the months and/or years before and after treatment (Figs. 1-12). The most common and persistent angiographic features of 24 months or more were summarized in Table 2.

\section{General Description of Vascular Characteristics of the Vascular-Avascular (V-Av) Junction and Features of Vascular Branching}

Before and after treatment, all the eyes, including the second line laser treated case, showed abnormal vascular branching at different levels of the vessels. An abnormal non-dichotomous, besom-like branching pattern was observed in almost all cases with some variations (Fig. 2). Irregular branching could be monitored at the large or small vessel level or at the precapillary level, and this irregularity could be detected in the same patient at all three levels simultaneously. In some cases, the branching pattern of the vessels showed sharper-angled branches, while in the others, more obliquely-angled branches were observed. In some patients, both features could be found at the same time and these features remained permanent (Fig. 2). Before the treatment, all the eyes demonstrated hyperfluorescence at the V-Av junction due to leakage. The most prominent features of FA taken before treatment were hyperfluorescence at the V-Av junction due to the vascular leakage, well-demarcated hyperfluorescent lesions, including focal capillary dilatations, cotton wool-like appearance as a vascular abnormality, capillary tufts in the capillary bed (usually appearing as "popcorn" abnormalities in fundus images), and arteriovenous shunts (Figs. 3-5 and 6). The rosary bead-like hyperfluorescent inside the vessels could be visible at the late venous phase of FA before and/or after the treatment (Fig. 7). After the treatment, strange anastomoses between the vessels and various vessel formations began to develop (Fig. 5). The circumferential vessel formation (also called naked arteriovenous shunt) that can run along the V-Av junction, were also observed frequently (Figs. 4 and 5). The retinal capillary network progress demonstrated developmental deficiencies both between existing vessels and between new developing vessels advancing into the retinal avascular space. Inadequacy of the capillary network system development and peculiar anastomoses were observed between large, medium and small vessels in most patients. These features were permanent in varying degrees for each patient (Fig.2). The persistence of avascular retina was a considerable angiographic feature observed at different rates in each patient. Although its severity continued to decrease gradually, vascular leaks that could continue to different degrees were observed in some cases. At older ages, FA revealed that irregular branching, bizarre anastomoses, and arteriovenous shunts were long-lasting features of the V-Av junction of all cases. Large tufts with tenuous leakage (Fig. 8), and besomlike branching with terminations including blunt capillary endings and zig-zag or circumferential shaped blunt anastomoses were detected. In all but one case (Fig. 9), retinal vascularization was not entirely completed by the conclusion of the observational period. Leakage patterns of developing vessels varied during long-lasting follow-up period on FA. The mottled leakage areas that occured in the late phases of FA in the V-Av junction were seen in older cases (Fig. 10). This observations suggested that the development of retinal vessels of treated premature infants may be a dynamic process continued over some years. Late recurrence did not develop in any patient due to the leakage or avascular areas.

\section{Features of the Vascularised Zone}

FA findings detected inside the vascularised zone comprised capillary nonperfusion and shunting throughout, areas of hypofluorescence and hyperfluorescence, leakage from vessels and massive loss of capillary bed around major vessels at the posterior pole and/or in the retina periphery (Fig. 11). Some of angiographic hypofluorescence areas were visible on ophthalmoscopy and RetCam images (Fig. 1), and some of these areas were surrounded by hyperfluorescent spots (Fig. 2). Small and/or large areas of retinal capillary obliteration and extensive loss of capillary bed were observed in vascularized retina in all cases in the pre-treatment phase and early period after the anti-VEGF therapy. Large hypofluorescent areas could be observed in such patients. These characteristics were observed as a persistent feature in many eyes with FA at older age (Fig. 10). A corkscrew-like linking shunt-vessel was observed between the main branching retinal vessels (Figs. 1 and 9). We noted this sign in almost all treated cases, irrespective of age. These lesions were inactive to angiography in the early and late phases of FA. Persistent tortuosity of the vessels was observed as a long-lasting feature of most of the cases (Figs. 9 and 10), furthermore, the level of tortuosity did not show any progressive change.

\section{Macular Abnormalities}

In the posterior pole and macular area, hypo- and hyperfluorescent lesions were observed in many patients. In Case 5, a large aberrant macular vessel connected with the regressed ridge was shown (Fig. 1). In the same patient, manifest pigmenter changes occured in macular area. At 3 years of age, there was a pigmented area that looked like a vertical retinal step in the macular area with a 2 OD diameter distance from the fovea (Fig. 1). At the pre-treatment phase and period soon after the treatment, we detected an absence of the foveal avascular zone (FAZ) in some cases. Although some vessels near the fovea narrowed this area (Figs. 9 and 10), FAZ developed in all cases.

\section{Choroidal Filling Pattern}

Irregular choroidal filling was observed in all eyes. Linear, lobular, and compound (linear-lobular) choroidal filling patterns on FA were recorded (Fig. 12). All three filling patterns were detected at all stages of the disease and at all ages of the patients. All three choroidal filling patterns are signs of immaturity of retinal and choroidal circulation. 


\section{Discussion}

During the past two decades, the use of intravitreal injections of anti-VEGF agents for the treatment of acute ROP has increased. The rapid regression of disease produced as a result of this treatment has understandably made it an attractive option for retina specialists. Due to the non-destructive nature of the treatment, intravitreal injection of anti-VEGF agents has been preferable to ablative therapies in some clinics. We aimed to report the early and late angiographic effects of intravitreal injection of bevacizumab and ranibizumab monotherapy in patients with Type 1 classical ROP and APROP. During the follow-up period, we observed the patients' various vascular development features. The presence of peripheral avascular retina is a finding that should be taken into consideration, as it may lead to subsequent complications. Because each patient's parental compliance was sufficient, we preferred to observe, rather than apply prophylactic LPT. We also aimed to present the effects of anti-VEGF treatment on developing retinas using FA from the pre-treatment period to early childhood and to discuss the results of our observational approach to retinal avascular areas.

Various studies have shown that FA should be considered a very useful and important instrument for monitoring period for ROP. Cernichiaro-Espinosa et al. emphasized that being a complex and insufficiently-understood process, vasculogenesis is being better studied with FA [13]. Al-Taie et al. investigated persistent avascular retina in infants with type 2 ROP which persisted after 45 weeks' PMA when regular ROP screening ceased [14]. Although they obtained fundus photographs during examination in order to reveal persistent peripheral avascular retina, they reported that the edge of the vascular/avascular border could be difficult to detect with this type of evaluation [14]. Lepore et al. also reported comprehensive studies on the importance of FA for diagnosis, treatment, and follow-up in the management of ROP $[11,15,16]$. Ho et al. emphasized that FA is advantageous owing to its ability to directly describe the vascularisation pattern of the retina, areas of avascular retina, and presence of any vessel leakage [17]. Purcaro et al. declared that IO alone is not the best diagnostic tool to identify ROP, but FA is safe and allows a more objective evaluation of the ROP stage and zone [18]. Ng et al. and Yokoi et al. also demonstrated that some vascular abnormalities could not be detected by IO, and FA allowed a superior observation of ROP disease [19, 20]. Nevertheless, multiple studies about the use of intravitreal anti-VEGF agents for acute ROP revealed full retinal vascular maturation without avascular areas formed after treatment, but the results were dependent on retinal examination and/or fundus photographs without FA imaging [7, 21-25]. In an earlier study, the authors reported successful results with ophthalmoscopically normal-appearing mature retina treated with IVB for acute ROP. The authors mentioned that the results of their study merely relied on 10 and did not include FA during a follow-up period, thus peripheral avascular zones could not be totally excluded based on long-term follow-up. The authors especially emphasized that this is an important weak point of their study [24]. In our opinion, IO is an absolutely gold standard screening exam for ROP, but it may be inadequate and controversial to argue that the retinal maturation is fully completed without retinal vascularisation being proved angiographically.

In recent years, multiple reports have been published about late recurrence after the intravitreal anti-VEGF treatment in ROP, which can lead to tractional retinal detachment (RD) and poor visual outcome [26-28]. The mechanism of late recurrence following intravitreal injection of anti-VEGF agents is still uncertain. Evaluation of anti-VEGF treated infants with FA allows the objective examination of retinal angiogenesis as emphasized previously. Tahija et al. [29] have reported on a series of both eyes of 10 babies who had been treated with IVB as sole therapy for Zone I and posterior Zone II up to 5 years. Their FA findings revealed that despite IVB's apparent effectiveness in bringing resolution of ROP and allowing growth of peripheral retinal vessels, complete normal peripheral retinal vascularization was not achieved in half of the patients [29]. Karkhaneh et al. reported a prospective clinical trial comparing the efficacy of LPT and IVB injection in infants with type 1 ROP in Zone II [30]. They concluded that the recurrence of neovascularization (NV), with IVB monotherapy was higher than that with conventional LPT, and the peripheral retina remains avascular for a long period after the injection; therefore, infants should be periodically monitored until the retina is entirely vascularized (sometimes up to 2 years) [30]. Toy et al. reported that full retinal maturation occurred in only $9 \%$ of IVB-treated eyes by 54 weeks' PMA. They hypothesized that treatment for ROP can be considered successful only with complete vascularization of the ora serrata [31]. It is obvious that some of the patients with ROP treated with anti-VEGF agents may be at high risk for late reactivation, even if the patient's follow-up is completed. At this point, which angiographic findings are important for prognosis of the disease and how to approach these cases who have abnormal vascularization characteristics on the retinal angiograms are key questions. These issues form the cornerstone of the present study. In our opinion, the primary consideration must be to clarify the importance of the presence of the lasting avascular retina and the significance of angiographic leakage. Will every remaining avascular retinal area be a threat in the future? A recent guideline suggests that examinations can be terminated in the following instances: PMA of 45 weeks and there is no worsening of ROP and no type 1 ROP disease. If anti-VEGF injectable medications were used to cause regression of the ROP, PMA of at least 65 weeks, then this treatment alters the natural history of this disease [32]. According to our practical observation with FA, not only our anti-VEGF treated cohort but also a considerable number of treatment-naive infants, who were followed without any prophylactic treatment, showed persistant avascular retinal area and different vascular abnormal features beyond 45 weeks, even into their toddler years (unpublished data). There is no consensus about the management of persistent avascular retina in ROP babies treated with antiVEGF agents, nor is there adequate data on untreated cases older than 45 weeks. In a prospective study, Warren et al. revealed peripheral avascular retina persisting beyond 45 weeks PMA in 16 infants with ROP using FA, 7 of whom had received IVB [33]. They highlighted the importance of continued surveillance beyond 50 weeks, especially in the context of prior treatment with IVB. They also concluded that although complications from persistent peripheral NV may be rare, LPT may be warranted to prevent late retinal complications [33], however, they have not applied this treatment to their cases. Ho et al. reported their experience with management of prematurely-born infants who did not fully vascularize after 45 weeks of PMA [17]. In these case series, 6 patients received prophylactic LPT for an avascular peripheral retina. However, these patients had evidence of peripheral NV, and/or ROP in Zone 3 with persistent plus disease in addition to the avascular retinal area [17]. They emphasized that peripheral NV may have been persistent NV that had not fully regressed or a new NV that resulted from peripheral nonperfusion. Since they were unable to determine the true etiology in their series, they also speculated that some of these patients may never fully vascularize, and a treating physician may elect to closely follow these cases without treatment, as there is no hard evidence currently supporting the opinion that LPT is superior or inferior [17]. In our cohort, we detected cases which had vascular leakage at V-Av junction on FA; nevertheless, if the acute phase of the disease had passed, then the disease had resolved, and the ridge had obviously begun to regress after the treatment. We concluded, therefore, that these findings were related to the immaturity of new, tiny, fragile, and developing vessels, thus, we chose 
not to apply LPT in these cases. During the follow-up period of these cases, vascular leakage significantly decreased or disappeared, but avascular retinal area remained at varying rates. Mansukhani et al. performed a retrospective study to report the FA findings at 60 weeks PMA and above after IVB for type 1 ROP and compared their findings for patients treated with IVB to untreated patients in whom ROP spontaneously regressed [34]. They administered LPT to both groups. The reasons for LTP were NV and persistent avascular retina in Zone II for IVB-treated group and solely presence of NV for untreated group. They believed that lesions with early hyperfluorescence and late leakage are indeed NV [34]. We considered that not all angiographic leakage are NV and endothelial cell dysfunction which is due to immaturity of new vessels may be the reason of leak, thus we preferred to follow this finding without an ablative approach. These leaks remained innocently for years in the vessels that could not complete their maturation entirely. We have not observed any aggravation and/or late complication related to this sign. Gonzalez et al. [35] aimed to compare reactivation rates and peripheral vascular findings on FA in classic ROP and APROP. They stated that their standard IVB treatment included planned, deferred prophylactic LPT to the residual avascular retina based on FA of the periphery, including the ora serrata region, at 60 weeks to 70 weeks of PMA, which they termed as 'treatment completion', in an attempt to reduce the risk of late reactivation of ROP [35]. They presented ocular outcomes after IVB with subsequent prophylactic treatment completion with LPT. They concluded that no RD was found in any eye that received IVB and prophylactic LPT [35]. However, by applying prophylactic laser to babies after 60 weeks, they could not observe in all cases whether their normal vascularization would progress or not. They also emphasized that the relatively long followup period of their study (range from 70 weeks to 211 weeks; mean, 125 weeks) makes the number of recurrences and late unfavorable structural outcomes more robust than previous studies with shorter follow-up, as reactivation can occur at up to 80 weeks or more [35]. Nonetheless, they could not evaluate whether complications would develop without laser application in the long term, thus in our opinion, their long follow-up period cannot give a clear idea of possible late complications of remaining avascular retina. Vural et al. compared to FA findings in type 1 and type 2 ROP with IVB monotherapy and spontaneous regression [36]. They performed FA at PMAs between 54 and 97 weeks in the type 1 ROP cases and between 43 and 86 weeks in the type 2 ROP babies. They emphasized that retinal vascularization can reach the farthermost limits in time without the need for an additional laser therapy for avascular retina, indicating importance of prolonged FA follow-up to prevent an unnecessary laser therapy. Nevertheless, contrary to our approach, the laser ablation was preferred by the authors to the avascular retina of babies who shown vascular leakage on FA during the early following period [36].

Mintz-Hittner and Kretzer reported in 1994 that prolonged retinal traction (by remnant shunt and EFP) between stable, posterior, prenatally vascularized retina, and unstable, postnatally vascularized retina may lead to the development of retinal holes characteristically located in the fragile, anterior, undifferentiated, nonvascularized retina [37]. In the present study, we also observed a late complication due to the remnant shunt and EFP in an infant (Case 5). Beyond the following period, the fundus examination of this patient showed a persistant ridge with regressed limited elevation, but vascular leakage at the V-Av border diminished gradually. At 104 weeks of PMA, the remnant ridge area showed elevation on IO examination, FA images showed marked vascular leakage at the same area. We treated this activation area with LPT, and subsequently it regressed. Snyder et al. reported a similar case of very late reactivation of ROP after IVB monotherapy was given for APROP [38]. At 2.5 years of uncorrected age for an infant who was born at 24 weeks of PMA, ROP reactivated in the form of tractional RD with vitreous hemorrhage in one eye and milder reactivation in the other. They emphasized that treatment with IVB may lead to chronic vascular arrest beyond standard screening guidelines of 54 weeks, posing a risk of late reactivation months to years later [38]. In another previous report, RD as late sequelae of ROP in a 19-year-old patient was presented [39]. The authors recommended that the late reactivation may due to persistent avascular retina generating continuous low levels of VEGF [39]. None of the aforementioned studies reported on FA findings of disease process after treatment without LPT. In our opinion, the suggested reasons of the disease reactivation for a few cases may speculative. In Case 5, laser treatment was planned due to constant ridge, however it could not perform due to refusal by the parents. Thus, we closely followed her for a long time and we detected remnant ridge at high reactivation risk with aberrant vessels extending from the macula; therefore, the persistently avascular area may not be a reason for very late reactivation. According to our judgement, if there are no other high-risk retinal abnormalities, the persistent avascular retina may not be a major risk factor alone. As mentioned before, possible constant production of VEGF by the peripheral avascular retina, which is thought to be the cause of very late complications, is a slow process. Numerous studies recommended that IVB-treated babies are at risk of chronic, life-long disease and require long-term observation with FA [38,39]. This also makes it possible to intervene immediately if a complication is detected. In our view, the key criterion is parental compliance with the follow-up schedule. If this is not possible, prophylactic LPT is inevitable. However, if the baby is routinely followed-up, it intervention can take place immediately if necessary. In the anti-VEGF era, where we are trying to avoid ablative treatment, how reasonable is it to apply prophylactic ablative treatment to babies whose risk factors we still cannot define? With this logic, we chose to follow our babies without ablative treatment and we did not encounter any late complications except from Case 5, who had the additional different risk factors mentioned earlier.

The importance of other persistent angiographic findings should also be considered for determination of the risk factors. According to our observations, most of the IVB-treated eyes had no regular vessel distribution with dichotomous pattern towards the periphery at a different stage. This aberrant vasculature was detected at three different levels in the present study: large, small, and precapillary. Lorenz et al. reported that in posterior Zone II disease and APROP, the abnormal branching events occurred at the small arteriolar level, while in Zone I disease, these branching events occurred almost exclusively at the large arteriolar level [40]. In contrast, in the present report, we observed that all types of vessel branching abnormalities were seen in all types of ROP disease. The circumferential vessels and strange forms of anastomosis were likewise permanently observed in almost all cases. Lepore et al. [15] observed a high rate of circumferential vessels before and after treatment and described it as a long-lasting finding of IVB treatment. This indication was consistent with our study result, and we do not consider this finding a reliable predictor of a possible late recurrence. The corkscrew-like linking shuntvessel is a common shunt formation between the branching retinal vessels. We believe that this is not an important permanent sign for late probable complications. It is our view that besom-like anomalous nondichotomous branching vascular terminals including immature vascular form, thus these primitive structures can show leakage on FA. Hence, after the maturation of vessels, which lasts from weeks to years, these leaked vascular endings may transform to blunted vessel terminals and shunts. Due to the permenant closing of the vascular terminals, the rest of retina may remain avascular. We propose that it is for this reason that the remnant avascular retinal area may not be ischemic and all leakage which can be observed on FA may not be due to the activation of ROP disease and/or NV. These areas should be followed. 
Although FA is not suitable for the evaluation of choroidal circulation, the present study revealed some features of the choroidal filling pattern. Lepore et al. evaluated the choroidal filling pattern before treatment and at age 4 years [16]. They emphasized that the presence of a linear choroidal pattern is associated with an immature choroid and retina vascular system, which persisted in bevacizumab-injected eyes at 4 years of age [16]. We observed the irregular choroidal filling patterns at a different ages of infants in the pretreatment and posttreatment period, even in the most advanced elderly children. Purcaro et al. [18] reported linear and lobular choroidal filling types, and emphasized that both are signs of extreme prematurity. In the present study, the persistence of irregularity in the choroidal filling pattern in older patients may be a marker of lasting immaturity of choroidal and retinal circulation. Flower hypothesized that the choroid has the potential to play a major role in maintaining the retina until the retinal vasculature fully develops [41]. Lepore et al. [11] concurred with this suggestion and mentioned such choroid and choriocapillary abnormalities, suggesting the need to examine the role of the choroid in the pathogenesis of functional and morphological damage of the retina, in particular the posterior pole. Although the choroidal findings of the study were limited, we agree with Lepore et al. New investigations on the choroidal filling pattern of premature infants may elucidate the maturation progress of premature infants.

In conclusion, the present study demonstrates that IVB and IVR can be very effective in the treatment of classic Type I ROP and APROP. Although IO is still the gold standard examination method, FA imaging is a very useful ancillary test both for monitoring vascular development and aid in determining risk factors in the vasculogenesis process that cannot be seen with routine examination by IO. In this study, although we have discussed several angiographic findings in infants treated with anti-VEGFs, the presence of leakage and remnant avascular retinal area may prove more likely than others to be possible risk factors for late relapses. We believe that every leak observed in the FA examination may not be evidence of activation, which may be related to vascular immaturity. In contrast to many studies in the literature, we did not implement laser therapy to those patients. This choice was made in light of the fact that we had performed intravitreal injection of anti-VEGF agents to protect the babies from laser related complications . In our judgement, prophylactic LPT for avascular retinal area may be redundant in most cases. In our opinion, due to the detected leakage patterns' change over time on FA, ROP disease may be a dynamic process progressing over several years, suggesting that babies with ROP should be monitored with decreasing frequency their whole life. Increased patient number and repeated visits may be a burden on physicians and parents, however, the cases already treated with ablative methods such as laser or cryotherapy should also be kept under surveillance. In situations where a medical center lacks FA, patients may be referred periodically to another clinic to be monitored. The significance of the other angiographic findings such as areas of hypofluorescence, the highest percentage of capillary bed loss, macular abnormalities, and lack of choroidal perfusion should be demonstrated through follow-up and studies to be performed over the time. Our observation approach may also be more daring than what is referred in the literature, and the safety of surveillance cannot be determined with so few cases. However, as Fierson stated, ROP care is developing, and suggestions may be modified as additional information about ROP risk factors, treatment results, and long-term outcomes are published [32]. An ongoing prospective study purposed to investigate the occurrence rates of an adverse prognosis including reactivation, retinal tear and RD after anti-VEGF therapy in ROP patients with persistent avascular retina; to assess the relationship between vascular leakage from FA results and the pathological changes. They aimed to compare the prognosis of persistent avascular retina with or without prophylactic peripheral photocoagulation in these patients; to shape the treatment strategy and provide evidence for the indications of prophylactic photocoagulation [42].

The limitations of the present study are the retrospective nature, the small number of cases, and the relatively short duration of follow-up. We hope that the findings of this study will contribute to the literature in the context of surveillance utilizing FA, and on the initiation of large, multicenter, controlled studies that will create new screening guidelines.

\section{Declarations}

None of the authors has conflict of interest with the submission

No financial support was received for this submission

The authors alone are responsible for the content and writing of the paper, specifically;

Conceptualization, data curation formal analysis investigation methodology project administration, visualization, writing - original draft : H.C.

Supervision, validation, writing - review \& editing: O.S.

Data availability: available, all data are stored

Consent to participate \& publish: there is no ethical problem.

\section{References}

1. Gilbert C, Rahi J, Eckstein M, O'Sullivan J, Foster A (1997) Retinopathy of prematurity in middle-income countries. Lancet 350: 12-14 Dol $10.1016 /$ S0140-6736(97)01107-0

2. Micieli JA, Surkont M, Smith AF (2009) A systematic analysis of the off-label use of bevacizumab for severe retinopathy of prematurity. Am J Ophthalmol 148: 536-543 e532 DOI 10.1016/j.ajo.2009.05.031

3. Boonstra N, Limburg H, Tijmes N, van Genderen M, Schuil J, van Nispen R (2012) Changes in causes of low vision between 1988 and 2009 in a Dutch population of children. Acta Ophthalmol 90: 277-286 DOI 10.1111/j.1755-3768.2011.02205.x

Page $7 / 19$ 
4. Faia LJ, Trese MT (2011) Retinopathy of prematurity care: screening to vitrectomy. Int Ophthalmol Clin 51: 1-16 DOI

$10.1097 /$ IIO.0b013e3182011033

5. (1988) Multicenter trial of cryotherapy for retinopathy of prematurity: preliminary results. Cryotherapy for Retinopathy of Prematurity Cooperative Group. Pediatrics 81: 697-706

6. Early Treatment For Retinopathy Of Prematurity Cooperative G (2003) Revised indications for the treatment of retinopathy of prematurity: results of the early treatment for retinopathy of prematurity randomized trial. Arch Ophthalmol 121: 1684-1694 D0I 10.1001/archopht.121.12.1684

7. Mintz-Hittner HA, Kennedy KA, Chuang AZ, Group B-RC (2011) Efficacy of intravitreal bevacizumab for stage 3+ retinopathy of prematurity. N Engl J Med 364: 603-615 DOI 10.1056/NEJMoa1007374

8. Wong RK, Hubschman S, Tsui I (2015) Reactivation of retinopathy of prematurity after ranibizumab treatment. Retina 35: 675-680 DOI 10.1097/IAE.0000000000000578

9. International Committee for the Classification of Retinopathy of P (2005) The International Classification of Retinopathy of Prematurity revisited. Arch Ophthalmol 123: 991-999 DOI 10.1001/archopht.123.7.991

10. (1984) An international classification of retinopathy of prematurity. The Committee for the Classification of Retinopathy of Prematurity. Arch Ophthalmol 102: 1130-1134 DOI 10.1001/archopht.1984.01040030908011

11. Lepore D, Molle F, Pagliara MM, Baldascino A, Angora C, Sammartino M, Quinn GE (2011) Atlas of fluorescein angiographic findings in eyes undergoing laser for retinopathy of prematurity. Ophthalmology 118: 168-175 DOI 10.1016/j.ophtha.2010.04.021

12. Blair MP, Shapiro MJ, Hartnett ME (2012) Fluorescein angiography to estimate normal peripheral retinal nonperfusion in children. J AAPOS 16: 234-237 DOI 10.1016/j.jaapos.2011.12.157

13. Cernichiaro-Espinosa LA, Olguin-Manriquez FJ, Henaine-Berra A, Garcia-Aguirre G, Quiroz-Mercado H, Martinez-Castellanos MA (2016) New insights in diagnosis and treatment for Retinopathy of Prematurity. Int Ophthalmol 36: 751-760 DOI 10.1007/s10792-016-0177-8

14. Al-Taie R, Simkin SK, Doucet E, Dai S (2019) Persistent Avascular Retina in Infants With a History of Type 2 Retinopathy of Prematurity: To Treat or Not to Treat? J Pediatr Ophthalmol Strabismus 56: 222-228 DOI 10.3928/01913913-20190501-01

15. Lepore D, Quinn GE, Molle F, Baldascino A, Orazi L, Sammartino M, Purcaro V, Giannantonio C, Papacci P, Romagnoli C (2014) Intravitreal bevacizumab versus laser treatment in type 1 retinopathy of prematurity: report on fluorescein angiographic findings. Ophthalmology 121 : $2212-2219$ DOI 10.1016/j.ophtha.2014.05.015

16. Lepore D, Quinn GE, Molle F, Orazi L, Baldascino A, Ji MH, Sammartino M, Sbaraglia F, Ricci D, Mercuri E (2018) Follow-up to Age 4 Years of Treatment of Type 1 Retinopathy of Prematurity Intravitreal Bevacizumab Injection versus Laser: Fluorescein Angiographic Findings. Ophthalmology 125: 218-226 DOI 10.1016/j.ophtha.2017.08.005

17. Ho LY, Ho V, Aggarwal H, Ranchod TM, Capone A, Jr., Trese MT, Drenser KA (2011) Management of avascular peripheral retina in older prematurely born infants. Retina 31: 1248-1254 DOI 10.1097/IAE.0b013e31820d3f70

18. Purcaro V, Baldascino A, Papacci P, Giannantonio C, Molisso A, Molle F, Lepore D, Romagnoli C (2012) Fluorescein angiography and retinal vascular development in premature infants. J Matern Fetal Neonatal Med 25 Suppl 3: 53-56 DOI 10.3109/14767058.2012.712313

19. Ng EY, Lanigan B, O'Keefe M (2006) Fundus fluorescein angiography in the screening for and management of retinopathy of prematurity. J Pediatr Ophthalmol Strabismus 43: 85-90

20. Yokoi T, Hiraoka M, Miyamoto M, Yokoi T, Kobayashi Y, Nishina S, Azuma N (2009) Vascular abnormalities in aggressive posterior retinopathy of prematurity detected by fluorescein angiography. Ophthalmology 116: 1377-1382 DOI 10.1016/j.ophtha.2009.01.038

21. Hwang CK, Hubbard GB, Hutchinson AK, Lambert SR (2015) Outcomes after Intravitreal Bevacizumab versus Laser Photocoagulation for Retinopathy of Prematurity: A 5-Year Retrospective Analysis. Ophthalmology 122: 1008-1015 DOI 10.1016/j.ophtha.2014.12.017

22. Harder BC, von Baltz S, Jonas JB, Schlichtenbrede FC (2014) Intravitreal low-dosage bevacizumab for retinopathy of prematurity. Acta Ophthalmol 92: 577-581 DOI 10.1111/aos.12266

23. Wu WC, Kuo HK, Yeh PT, Yang CM, Lai CC, Chen SN (2013) An updated study of the use of bevacizumab in the treatment of patients with prethreshold retinopathy of prematurity in taiwan. Am J Ophthalmol 155: 150-158 e151 DOI 10.1016/j.ajo.2012.06.010

24. Yetik H, Gunay M, Sirop S, Salihoglu Z (2015) Intravitreal bevacizumab monotherapy for type-1 prethreshold, threshold, and aggressive posterior retinopathy of prematurity - 27 month follow-up results from Turkey. Graefes Arch Clin Exp Ophthalmol 253: 1677-1683 DOI 10.1007/s00417-014-2867-0 
25. Klufas MA, Patel SN, Ryan MC, Patel Gupta M, Jonas KE, Ostmo S, Martinez-Castellanos MA, Berrocal AM, Chiang MF, Chan RV (2015) Influence of Fluorescein Angiography on the Diagnosis and Management of Retinopathy of Prematurity. Ophthalmology 122: 1601-1608 DOI

10.1016/j.ophtha.2015.04.023

26. Hu J, Blair MP, Shapiro MJ, Lichtenstein SJ, Galasso JM, Kapur R (2012) Reactivation of retinopathy of prematurity after bevacizumab injection. Arch Ophthalmol 130: 1000-1006 DOI 10.1001/archophthalmol.2012.592

27. Patel RD, Blair MP, Shapiro MJ, Lichtenstein SJ (2012) Significant treatment failure with intravitreous bevacizumab for retinopathy of prematurity. Arch Ophthalmol 130: 801-802 DOI 10.1001/archophthalmol.2011.1802

28. Ittiara S, Blair MP, Shapiro MJ, Lichtenstein SJ (2013) Exudative retinopathy and detachment: a late reactivation of retinopathy of prematurity after intravitreal bevacizumab. J AAPOS 17: 323-325 DOI 10.1016/j.jaapos.2013.01.004

29. Tahija SG, Hersetyati R, Lam GC, Kusaka S, McMenamin PG (2014) Fluorescein angiographic observations of peripheral retinal vessel growth in infants after intravitreal injection of bevacizumab as sole therapy for zone I and posterior zone II retinopathy of prematurity. Br $\mathrm{J}$ Ophthalmol 98 : 507-512 DOI 10.1136/bjophthalmol-2013-304109

30. Karkhaneh R, Torabi H, Khodabande A, Roohipoor R, Riazi-Esfahani M (2018) Efficacy of Intravitreal Bevacizumab for the Treatment of Zone I Type 1 Retinopathy of Prematurity. J Ophthalmic Vis Res 13: 29-33 DOI 10.4103/jovr.jovr_198_16

31. Toy BC, Schachar IH, Tan GS, Moshfeghi DM (2016) Chronic Vascular Arrest as a Predictor of Bevacizumab Treatment Failure in Retinopathy of Prematurity. Ophthalmology 123: 2166-2175 DOI 10.1016/j.ophtha.2016.06.055

32. Fierson WM, Ophthalmology AAOPSo, American Academy Of O, American Association For Pediatric O, Strabismus, American Association Of Certified O (2018) Screening Examination of Premature Infants for Retinopathy of Prematurity. Pediatrics 142 DOI 10.1542/peds.2018-3061

33. Warren CC, Young JB, Goldberg MR, Connor TB, Kassem IS, Costakos DM (2018) Findings in Persistent Retinopathy of Prematurity. Ophthalmic Surg Lasers Imaging Retina 49: 497-503 DOI 10.3928/23258160-20180628-05

34. Mansukhani SA, Hutchinson AK, Neustein R, Schertzer J, Allen JC, Hubbard GB (2019) Fluorescein Angiography in Retinopathy of Prematurity: Comparison of Infants Treated with Bevacizumab to Those with Spontaneous Regression. Ophthalmol Retina 3: 436-443 DOI 10.1016/j.oret.2019.01.016

35. Garcia Gonzalez JM, Snyder L, Blair M, Rohr A, Shapiro M, Greenwald M (2018) Prophylactic Peripheral Laser and Fluorescein Angiography after Bevacizumab for Retinopathy of Prematurity. Retina 38: 764-772 DOI 10.1097/IAE.0000000000001581

36. Vural A, Ekinci DY, Onur IU, Hergünsel GO, Yiğit FU. (2019) Comparison of fluorescein angiographic findings in type 1 and type 2 retinopathy of prematurity with intravitreal bevacizumab monotherapy and spontaneous regression. Int Ophthalmol. 39(10):2267-2274. doi: 10.1007/s10792-018-010647.

37. Mintz-Hittner HA, Kretzer FL (1994) Postnatal retinal vascularization in former preterm infants with retinopathy of prematurity. Ophthalmology 101: 548-558 DOI 10.1016/s0161-6420(94)31301-7

38. Snyder LL, Garcia-Gonzalez JM, Shapiro MJ, Blair MP (2016) Very Late Reactivation of Retinopathy of Prematurity After Monotherapy With Intravitreal Bevacizumab. Ophthalmic Surg Lasers Imaging Retina 47: 280-283 DOI 10.3928/23258160-20160229-12

39. Golas L, Shapiro MJ, Blair MP (2018) Late ROP Reactivation and Retinal Detachment in a Teenager. Ophthalmic Surg Lasers Imaging Retina 49: 625-628 DOI 10.3928/23258160-20180803-11

40. Lorenz B, Stieger K, Jager M, Mais C, Stieger S, Andrassi-Darida M (2017) RETINAL VASCULAR DEVELOPMENT WITH 0.312 MG INTRAVITREAL BEVACIZUMAB TO TREAT SEVERE POSTERIOR RETINOPATHY OF PREMATURITY: A Longitudinal Fluorescein Angiographic Study. Retina 37: 97-111 DOI 10.1097/IAE.0000000000001126

41. Flower R (1985) Perinatal retinal vascular physiology. Blackwell Sci. Publ.; , Boston

42. Yu Y, Wang J, Chen F, Chen W, Jiang N, Xiang D (2020) Study protocol for prognosis and treatment strategy of peripheral persistent avascular retina after intravitreal anti-VEGF therapy in retinopathy of prematurity. Trials 21: 493 DOI 10.1186/s13063-020-04371-6

\section{Tables}

\section{Table 1. General characteristics of patients}




\begin{tabular}{|c|c|c|c|c|c|c|c|c|c|c|c|c|}
\hline $\begin{array}{c}\text { Case } \\
\text { Number }\end{array}$ & $\begin{array}{c}\text { GA } \\
\text { (Weeks) }\end{array}$ & $\begin{array}{c}\text { Birth } \\
\text { Weight } \\
\text { (g) }\end{array}$ & Gender & $\begin{array}{l}\text { ROP } \\
\text { Stage }\end{array}$ & $\begin{array}{c}\text { Plus } \\
\text { Disease }\end{array}$ & Eye & $\begin{array}{l}\text { Anti- } \\
\text { VEGF }\end{array}$ & $\begin{array}{l}\text { Tx Time } \\
\text { (Weeks) }\end{array}$ & $\begin{array}{c}\text { Presence } \\
\text { of } \\
\text { Recurrence }\end{array}$ & $\begin{array}{c}\text { Recurrence } \\
\mathrm{Tx}\end{array}$ & $\begin{array}{c}\text { Complete } \\
\text { retinal vascularization } \\
\text { (Weeks) } \\
\text { (Exam and FA) }\end{array}$ & $\begin{array}{l}\text { Follow- } \\
\text { Up Time } \\
\text { (Months) }\end{array}$ \\
\hline 1 & 31,0 & 1580 & M & Z1/S3 & + & $\mathrm{OU}$ & B & 36,0 & - & - & $\begin{array}{c}\text { Exam: Completed at } \\
50^{\text {th }} \text { week } \\
\text { FA: Incomplete }\end{array}$ & 71 \\
\hline 2 & 25,1 & 760 & $\mathrm{~F}$ & Z2p/S3 & + & $\mathrm{OU}$ & B & 38,1 & - & - & $\begin{array}{c}\text { Exam: Completed at } \\
47^{\text {th }} \text { week } \\
\text { FA: Incomplete }\end{array}$ & 71 \\
\hline 3 & 24,5 & 555 & $\mathrm{~F}$ & Z2p/S3 & + & OS & B & 32,5 & - & - & $\begin{array}{c}\text { Exam: Completed at } \\
44^{\text {th }} \text { week } \\
\text { FA: OD: Completed at } \\
168^{\text {th }} \text { week } \\
\text { OS: Incomplete }\end{array}$ & 69 \\
\hline 4 & 25,1 & 720 & $\mathrm{~F}$ & Z1/S3 & Preplus & $\mathrm{OU}$ & B & 35,1 & - & - & $\begin{array}{c}\text { Exam: Completed at } \\
55^{\text {th }} \text { week } \\
\text { FA: Completed at } \\
108^{\text {th }} \text { week }\end{array}$ & 60 \\
\hline 5 & 24,0 & 845 & $\mathrm{~F}$ & Z1/S3 & + & $\mathrm{OU}$ & B & 32,0 & $\begin{array}{l}\text { First: } \\
1^{\text {st }} \text { month } \\
\text { of tx } \\
\text { Second: At } \\
104 \text { weeks } \\
\text { of tx }\end{array}$ & $\begin{array}{l}\text { First: } \\
\text { Second } \\
\text { injection of } \\
\text { B } \\
\text { Second: } \\
\text { Laser tx }\end{array}$ & $\begin{array}{c}\text { Exam: Completed at } \\
54^{\text {th }} \text { week* }^{*} \\
\text { FA: Completed at } \\
96^{\text {th }} \text { week* }\end{array}$ & 57 \\
\hline 6 & 28,0 & 900 & $\mathrm{~F}$ & Z1/S3 & Preplus & $\mathrm{OU}$ & B & 36,0 & - & - & $\begin{array}{c}\text { Exam: Completed at } \\
50^{\text {th }} \text { week } \\
\text { FA: Incomplete }\end{array}$ & 50 \\
\hline 7 & 26,3 & 1006 & $\mathrm{~F}$ & $\mathrm{Z} 2 \mathrm{p} / \mathrm{S} 3$ & + & OD & B & 42,3 & - & - & $\begin{array}{c}\text { Exam: Completed at } \\
52^{\text {th }} \text { week } \\
\text { FA: Incomplete }\end{array}$ & 59 \\
\hline $8^{\dagger}$ & 23,0 & 790 & $\mathrm{M}$ & $\mathrm{Z2p} / \mathrm{S3}$ & + & OD & B & 33,0 & $\begin{array}{c}\text { At } 8 \text { weeks } \\
\text { of tx }\end{array}$ & Laser tx & Ablated with laser & 72 \\
\hline 9 & 27,0 & 620 & $\mathrm{M}$ & $\mathrm{Z2p} / \mathrm{S3}$ & + & OU & $\mathrm{R}$ & 41,0 & - & - & $\begin{array}{c}\text { Exam: Completed at } \\
67^{\text {th }} \text { week } \\
\text { FA: Incomplete }\end{array}$ & 32 \\
\hline 10 & 26,5 & 605 & $\mathrm{M}$ & APROP & + & $\mathrm{OU}$ & $\mathrm{R}$ & 36,5 & - & - & $\begin{array}{c}\text { Exam: Completed at } \\
56^{\text {th }} \text { week } \\
\text { FA: Incomplete }\end{array}$ & 32 \\
\hline 11 & 25,6 & 730 & $\mathrm{~F}$ & $\mathrm{Z} 2 \mathrm{p} / \mathrm{S} 3$ & + & $\mathrm{OU}$ & $\mathrm{R}$ & 37,6 & - & - & $\begin{array}{c}\text { Exam: Completed at } \\
71^{\text {th }} \text { week } \\
\text { FA: Incomplete }\end{array}$ & 24 \\
\hline 12 & 27,6 & 885 & $\mathrm{M}$ & Z2p/S3 & + & OS & $\mathrm{R}$ & 39,6 & - & - & $\begin{array}{c}\text { Exam: Completed at } \\
90^{\text {th }} \text { week } \\
\text { FA: Incomplete }\end{array}$ & 24 \\
\hline 13 & 28,0 & 850 & $\mathrm{~F}$ & Z2p/S3 & + & $\mathrm{OU}$ & B & 39,0 & - & - & 48 & $6 * *$ \\
\hline 14 & 27,0 & 880 & M & $\mathrm{Z} 2 \mathrm{p} / \mathrm{S} 3$ & + & OS & B & 36,0 & - & - & 51 & $44 * * *$ \\
\hline 15 & 27,0 & 970 & $\mathrm{M}$ & $\mathrm{Z2p/S3}$ & + & OS & B & 35,0 & - & - & 52 & $44 * * *$ \\
\hline 16 & 24,0 & 575 & M & Z2p/S3 & + & $\mathrm{OU}$ & B & 34,0 & - & - & Died & 4 \\
\hline $17^{t t}$ & 26,0 & 850 & $\mathrm{~F}$ & $\begin{array}{l}\text { APROP/ } \\
\mathrm{Z} 2 \mathrm{p} / \mathrm{S} 3\end{array}$ & + & OU & B & $38,0 / 42,0$ & $\begin{array}{l}\text { At } 1 \\
{ }^{\text {st }} \text { month of } \\
\text { tx } \\
\text { At } \\
42^{\text {th }} \text { week } \\
\text { of birth OS }\end{array}$ & $\begin{array}{l}\text { Second } \\
\text { injection of } \\
\text { half dose } \\
\text { of B }\end{array}$ & Incomplete & 15 \\
\hline
\end{tabular}


was

treated

firstly

\begin{tabular}{|c|c|c|c|c|c|c|c|c|c|c|c|c|}
\hline $18^{t \dagger}$ & 23,6 & 660 & $\mathrm{~F}$ & $\mathrm{Z} 2 \mathrm{p} / \mathrm{S} 3$ & + & OR & B & 35,4 & - & - & Incomplete & 11 \\
\hline $19^{t t}$ & 26,0 & 1135 & $\mathrm{~F}$ & Z1/S3 & + & OU & $\mathrm{B}$ & 36,5 & - & - & Incomplete & 10 \\
\hline
\end{tabular}

APROP: Aggressive posterior retinopathy of prematurity, B: Bevacizumab, Exam: Examination with binocular ophthalmoscopy, F: Female, FA: Fundus Fluorescein Angiography, GA: Gestational Age, M: Male, OD: Oculus Dexter (right eye), OS: Oculus Sinister (left eye), OU: Oculus Uterque (both eyes), R: Ranibizumab, ROP: Retinopathy of prematurity, Tx: Treatment, VEGF: Vascular endothelial growth factor, Z1/S3: Zone 1/Stage 3, Z2p/S3: Zone 2 posterior/Stage 3

$+\quad$ treated with laser due to the refusal of the parents of bevacuzimab

tt incomplete in both examinations (binocular ophthalmoscopy and fundus fluorescein angiography)

* except persistent elevation of temporal quadrant in Oculus Dexter

** after $6^{\text {th }}$ month-out of follow-up, an immigrant patient, following at Syria

*** after $44^{\text {th }}$ month- out of follow-up, due to moving another country in Turkey

Table 2. The number and percentage of eyes with the most common persistent angiographic features $(n=19) *$

Feature Number of Latest FA Percentage

\begin{tabular}{lcc}
\hline Abnormal non-dichotomous, besom-like branching pattern & 19 & 100 \\
\hline Peripheral avascular area. & 17 & 89.5 \\
\hline Vascular leakage. & 11 & $58^{* *}$ \\
\hline Naked arteriovenous shunt & 13 & 68 \\
\hline Macular Abnormalities & 8 & 42 \\
\hline Irregular choroidal filling & 19 & 100 \\
& & 31.6 \\
\hline Mottled leakage areas at the late phase & 6 & \\
\hline
\end{tabular}

* the late term findings of cases, older than 24 months

** from mild to moderate, in variable degrees

\section{Figures}




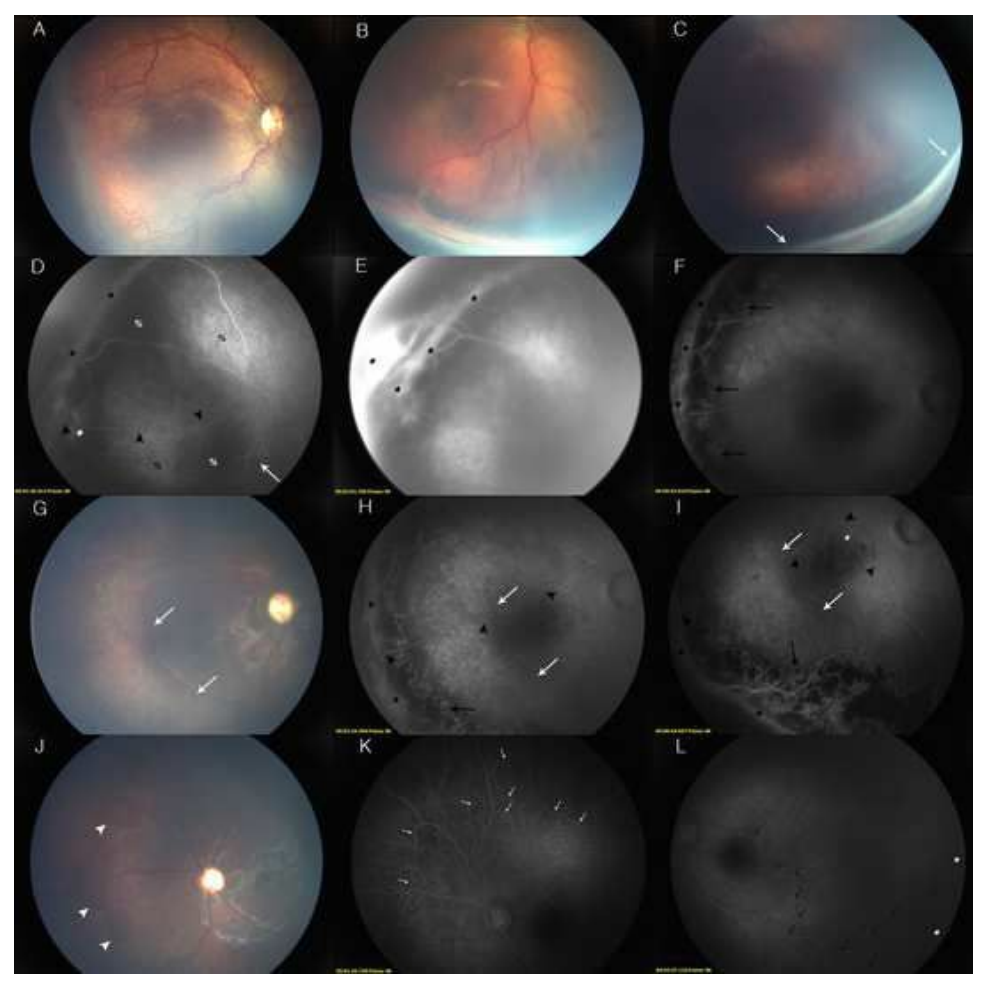

Figure 1

RetCam color fundus photographs (A-C) and fluorescein angiography images (D-L) belonging to Case 5 . The images represent respectively: (A), before the first injection, (B), before the second injection, (C), one month after the second injection, (D), first year of follow-up period, (E), third activation at 26 months, $(F)$, one month after the laser treatment, (G-L), at age of 5 years. C, limited regressing EFP (white arrows). The family did not allow laser treatment, thus the patient was monitored periodically. D, residue EFP with limited FL at the V-Av J (black asterisks), dotted hyperfluorescence in the macular area (white arrow) and posterior of the remnant EFP (white asterisk) due to pigmentary changes, large aberrant vessel between regressed ridge and fovea (black arrowheads), dispersed hyperfluorescent and hypofluorescent areas in vascularized retina (black and white percent sign, respectively). E, significant FL and retinal elevation at the V-Av J (black asterisks). F, marked regression of FL at V-Av J after laser treatment (black asterisks), laser spots (black arrows). G-I, step-like appearance of pigmentary changes of macula (white arrows), persistent limited FL at the V-Av J (black asterisks), laser spots (black arrows), large abnormal shunt vein associated with the ridge originating from the foveal area (black arrowheads), disorganization of the FAZ (white asterisk). $\mathrm{J}$ and $\mathrm{K}$, large hyperpigmented areas (white arrowheads), lesions that did not show any feature angiographically, corkscrew-like anastomoses (small white arrows). $\mathrm{L}$, tortuous shunt vessels crossing the fovea in the posterior pole (small black arrows), shunt vessels progressing to the periphery in the form of besom-like branching (small black arrows), peripheral avascular retina (white asterisks). extraretinal fibrovascular proliferation: EFP, fluorescein leakage: FL, foveal avascular zone: FAZ, vascular-avascular junction: V-Av J

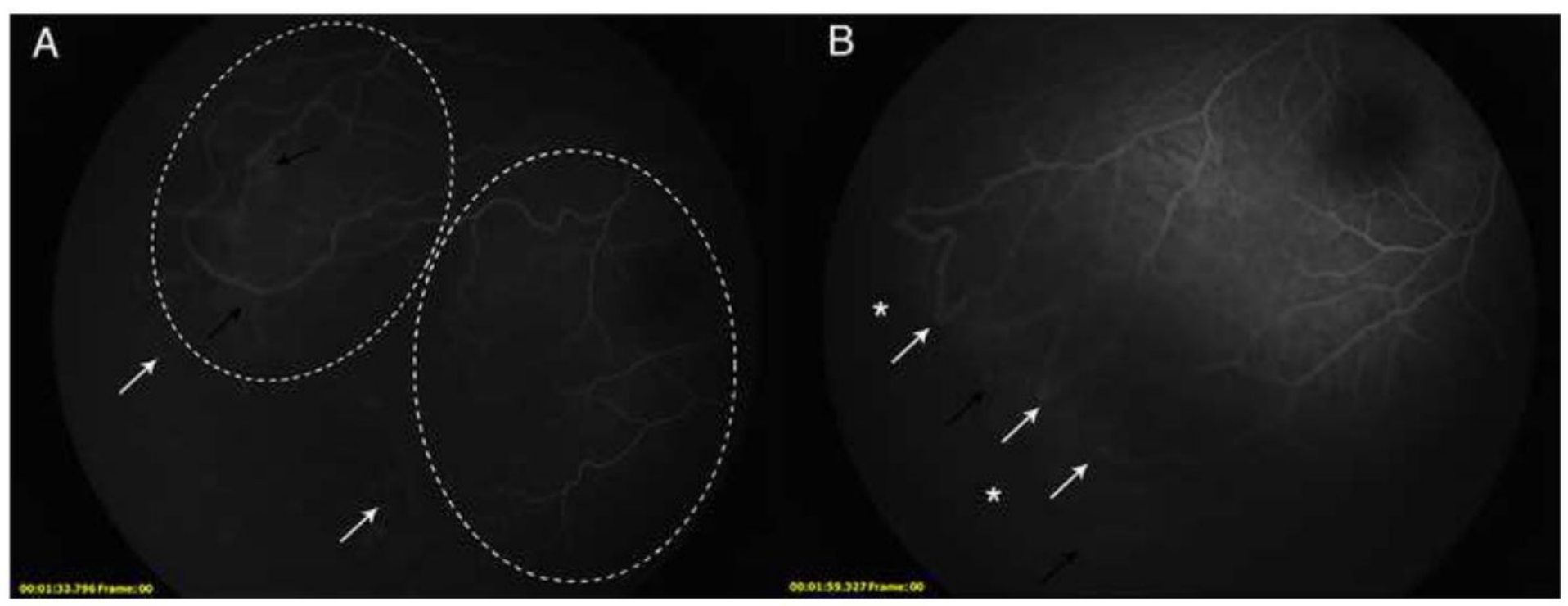

Figure 2 
Montage fluorescein angiography images of 6-year-old preterm child who received bevacizumab treatment to the left eye (A-E). The signs of developmental abnormalities are observed not only in the treated eye, but also in the untreated right eye. A and B, besom-like anomalous non-dichotomous branching pattern in both eyes (similar to besom image below), A, areas with (black arrows) and without (white arrow) capillary network between the vessels. B and D, persistent large avascular peripheral retina, without leakage (white asterisks) and with indistinct leak (black asterisks). (B-E), various branching anomalies at different levels of vessels; sharp-angled, elbowed, peripheral branching pattern (black arrows) and soft-shaped branching pattern (white arrows) near the posterior pole. C, pigmentary dystrophy area (white arrowhead).

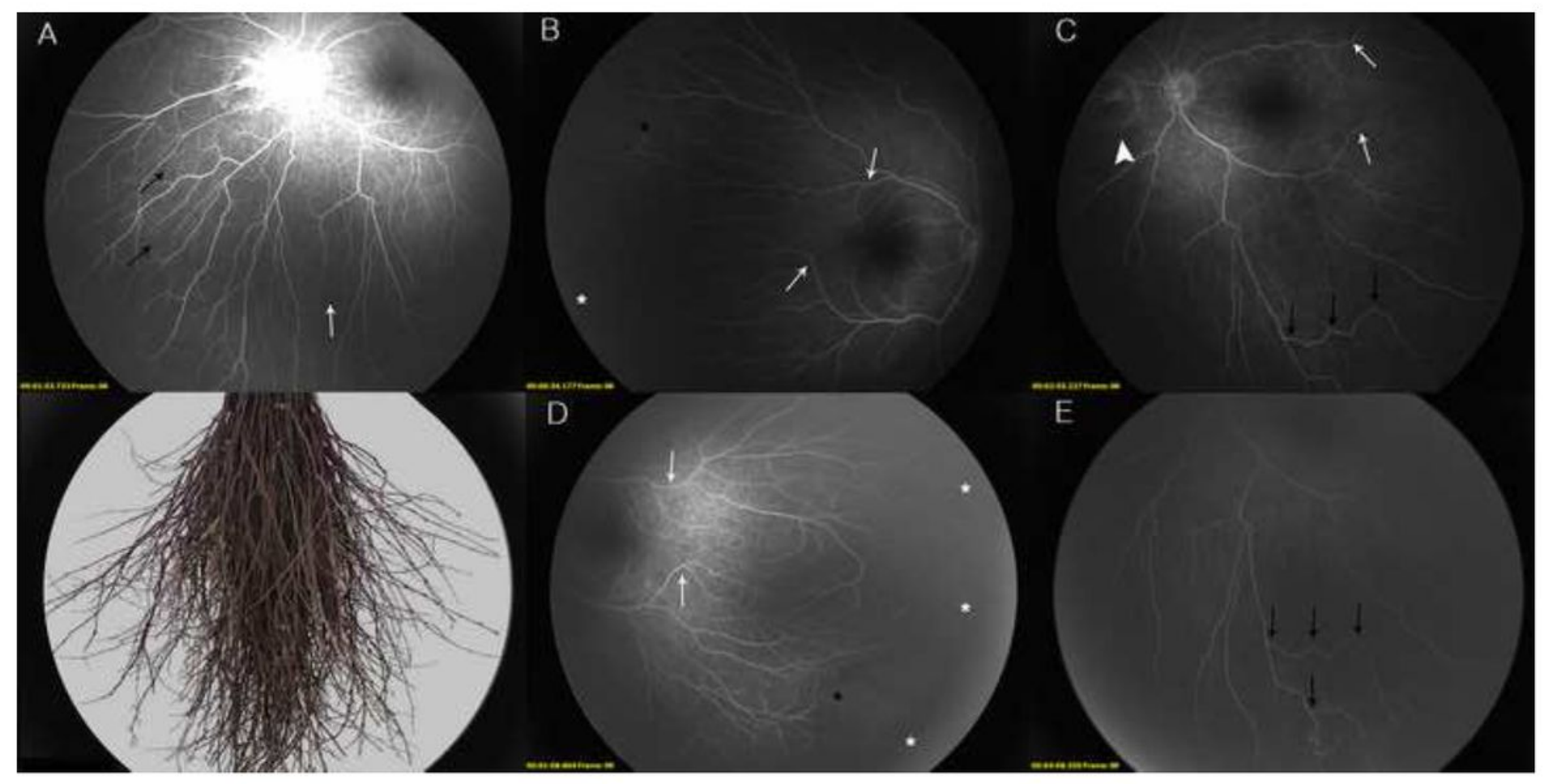

\section{Figure 3}

Angiographic image showing the highly-immature retina of a baby with aggressive posterior ROP, before treatment. Hyperfluorescence at the vascularavascular junction due to the vascular leakage (black asterisks), focal capillary dilatations (black arrows), capillary tufts in the capillary bed (white arrow), arteriovenous shunts (black dotted circle), and haemorrhage (white asterisk). 


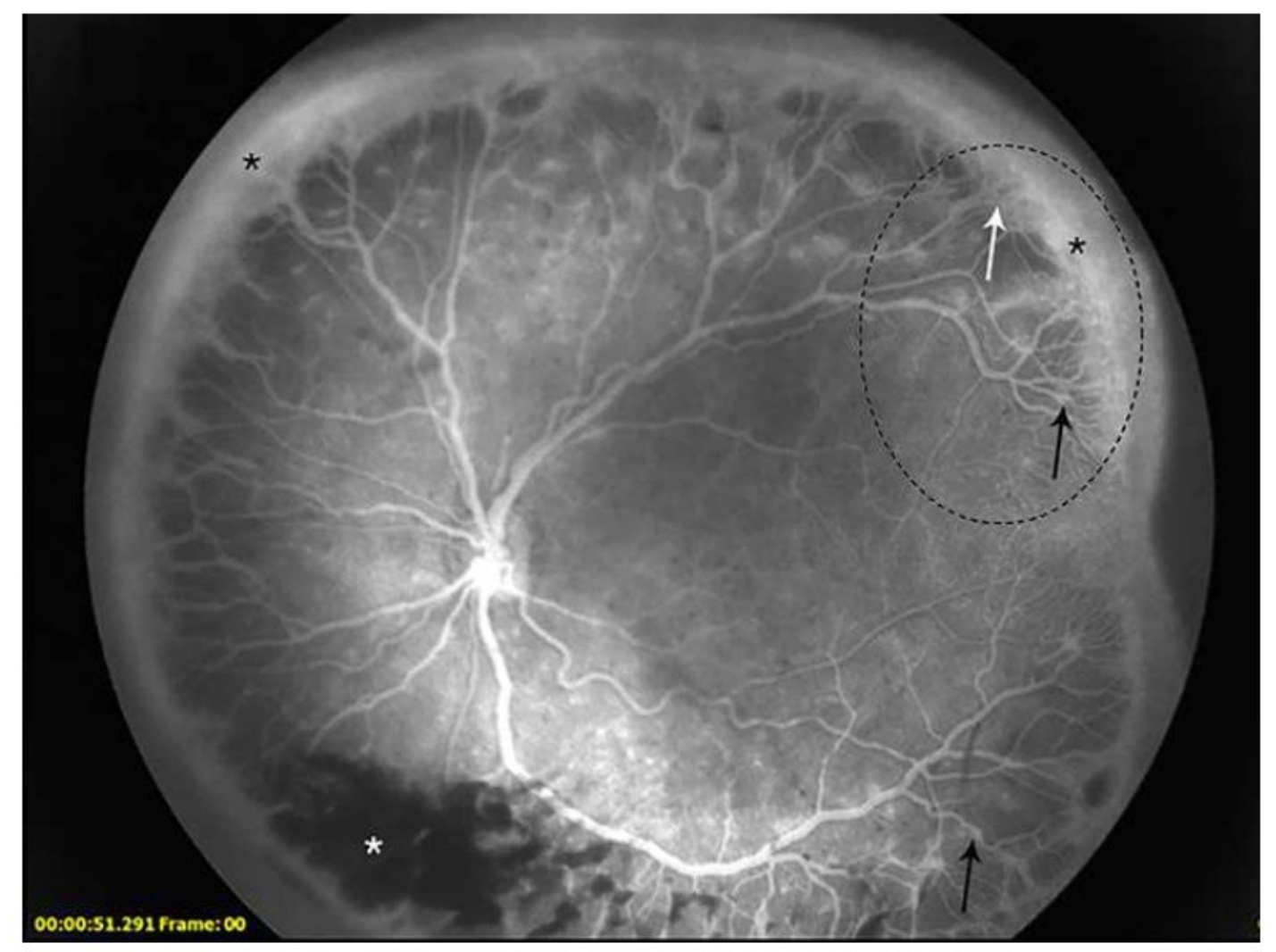

\section{Figure 4}

Montage images of a 3-year-old patient. The images represent respectively: (A and D), before treatment, (B and $E)$, one month after treatment, (C and F) 6 months after treatment, and (G-I), 30 months after treatment. RetCam color fundus photographs (A, B) and fluorescein angiography images (D, E), the locations of the findings corresponding to the lesions are numbered with white arrows in both; 1 . leakage of fluorescein from neovascularization at the vascular-avascular (V-Av) junction, 2. focal dilation of capillaries, 3. capillary tuft formation, 4. hyperfluorescent lesions and diffuse perivascular leakage near the V-Av J. C and F, circumferential vessel (naked arteriovenous shunt) (black arrows), tiny loops (black asterisks), sharp-angled, elbowed branched vessels (white arrowheads) extending to the retinal periphery with besom-like vascularisation (black arrowheads). (G-I), persistent avascular peripheral retina (white asterisks) with and without leakage (black arrows) in V-Av J. vascular-avascular junction: V-Av J 


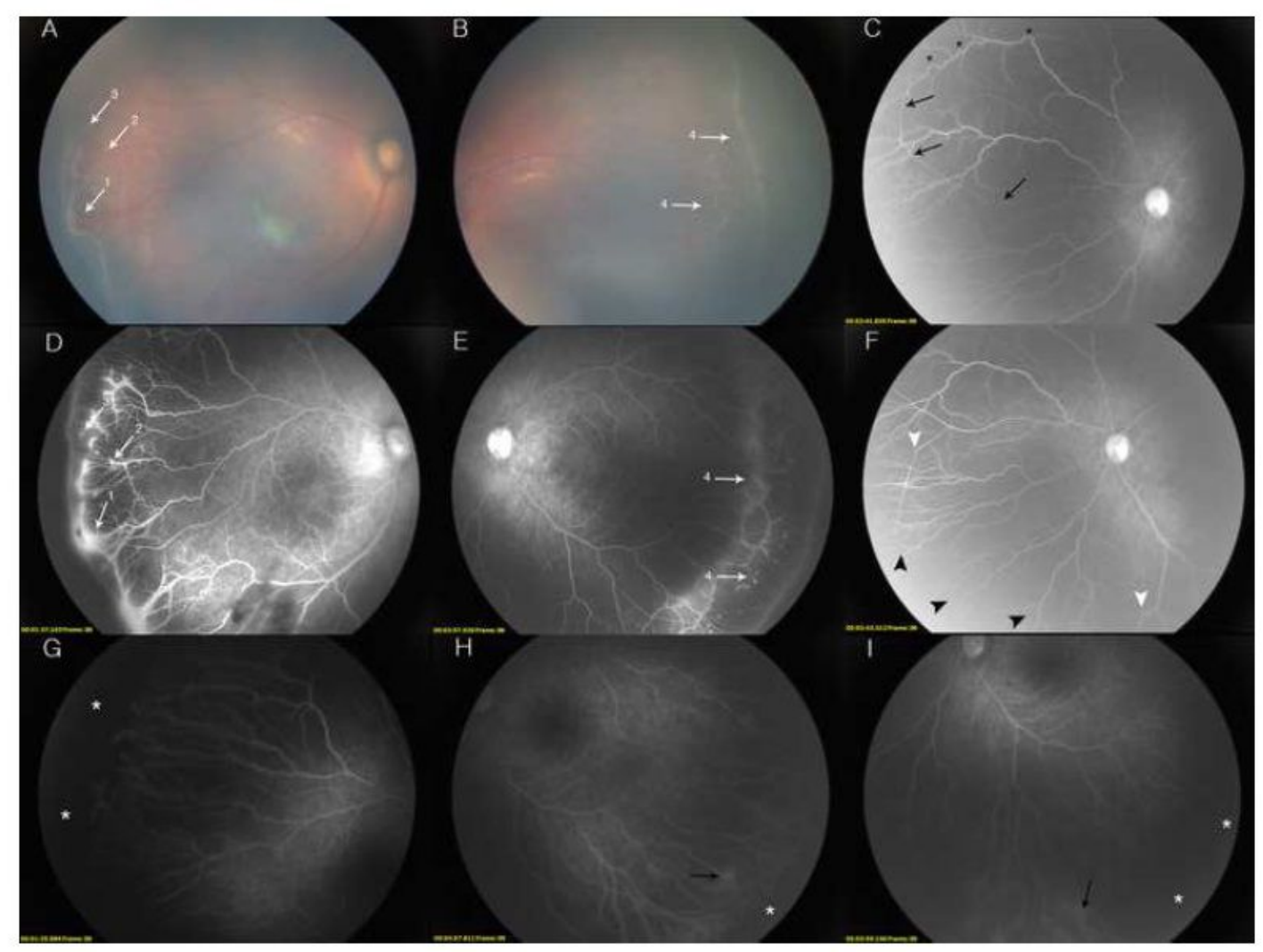

\section{Figure 5}

Fluorescein angiography images of 3 different preterm children (A-D, E-H, I-L figures belong to those respectively). (A,E,I,C,G,K) images, before treatment, FL from NV at the V-Av J (black asterisk), focal dilation of capillaries (black arrows), capillary tuft formation next to the $\mathrm{V}$-Av $\mathrm{J}$ (white arrows), hyperfluorescent lesions with cotton wool-like appearance near the $\mathrm{V}-\mathrm{Av} \mathrm{J}$ (black arrowheads), elevation of large vessels at the nasal side (white arrowheads). (B,F,J,D,H,L) images, 6th month after treatment, (B), EFP completely regressed, (D), retinal elevation flattened (black arrowheads). (F,H, J, L), large avascular peripheral retina (white asterisks), hyperfluorescent and hypofluorescent areas in vascularized retina (black and white percent signs, respectively), weak leakage at $\mathrm{V}$ $A v \mathrm{~J}$ (black arrows), blunted vessel endings (white arrowheads). (F), strangely-shaped circumferential vessel formation (white arrow), capillary tufts beyond naked shunt vessels at V-Av J with slight leakage (black arrowheads). extraretinal fibrovascular proliferation: EFP, fluorescein leakage: FL, foveal avascular zone: FAZ, NV: neovascularization, vascular-avascular junction: V-Av J

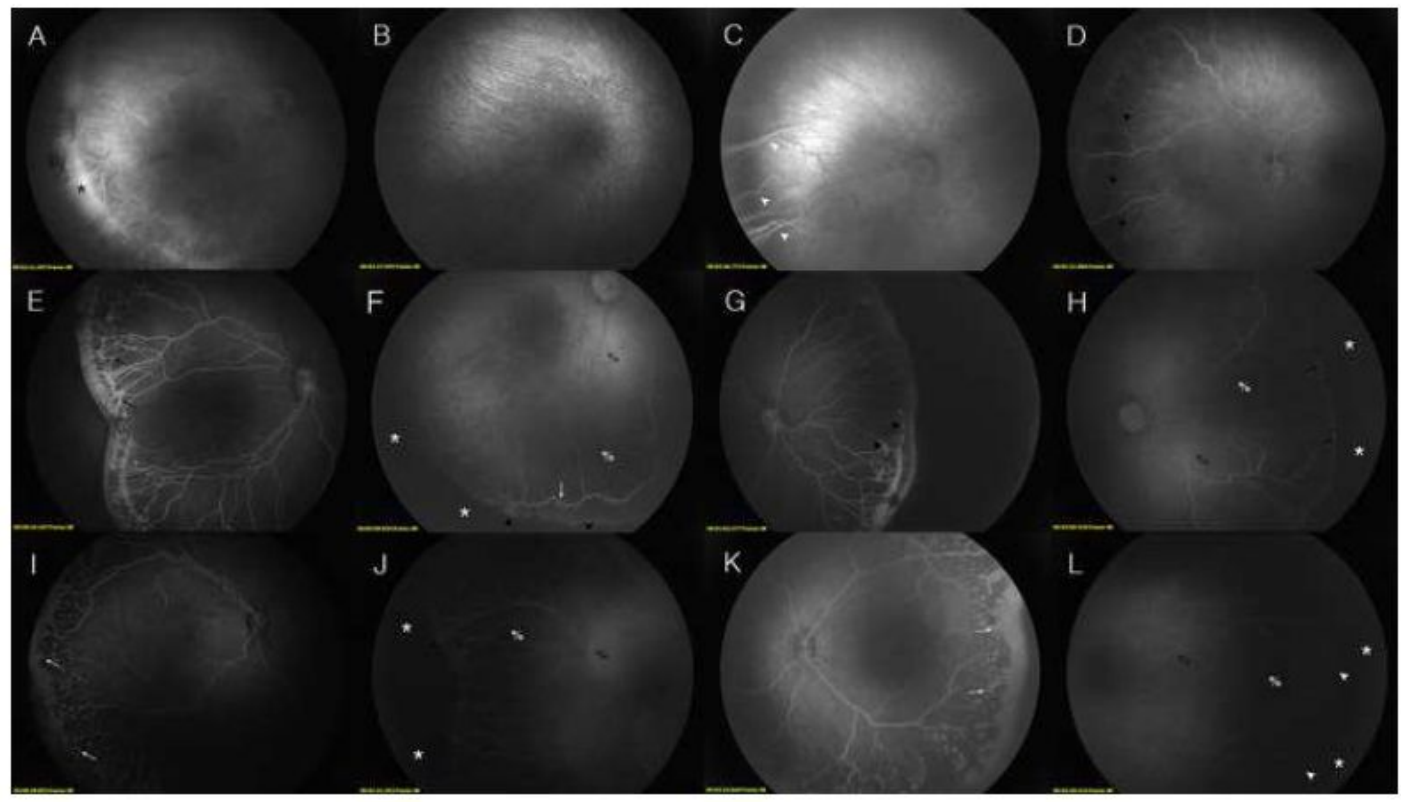

Figure 6 
RetCam color fundus photographs (A and G) and FA images (B-F, H-L) belonging to a 2-year-old patient; (A-F, K, L) images related to the right eye, (G-J) images related to the left eye. A and B, before treatment, FL from NV at the V-Av J (black asterisks), focal dilatation of capillaries (black arrow), (C), 6 months after treatment, PAPR (white asterisks), large and capillary tufts with leakage at V-Av J (white arrows), marked leakage zone in vascularized retina (black arrow), (D-E), one year after treatment, PAPR (white asterisks), irregular branching in all levels of the vessels with anomalous shunts (black arrows), significant leakage from immature vascularization at early and late phase of FA (white arrows). (F), 2 years after treatment, since the maturation of the vessels was not completed, the leak continued (white arrows), PAPR (white asterisks). G and H, before treatment, FL from NV at the V-Av J (black asterisks), focal dilation of capillaries (black arrows), capillary tuft formation next to the $\mathrm{V}$-Av $\mathrm{J}$ (white arrows), hyperfluorescent lesions with cotton wool-like appearance near the V-Av J (white arrowhead), I, 6 months after treatment, PAPR (white asterisks), abnormal besom-like non-dichotomous branching with strange anastomosis (black dotted circle) and blunted vessel terminals with slight FL (black arrows), J, 2 years after treatment, PAPR (white asterisks), blunted vessel terminals without leakage (black arrows), $\mathrm{K}$ and $\mathrm{L}$, one year and 2 years after treatment, respectively, PAPR (white asterisks), with FL resulting from the immaturity of vessels (white arrows). extraretinal fibrovascular proliferation: EFP, fluorescein angiography: FA, fluorescein leakage: FL, foveal avascular zone: FAZ, NV: neovascularization, PAPR: persistent avascular peripheral retina, vascular-avascular junction: V-Av J

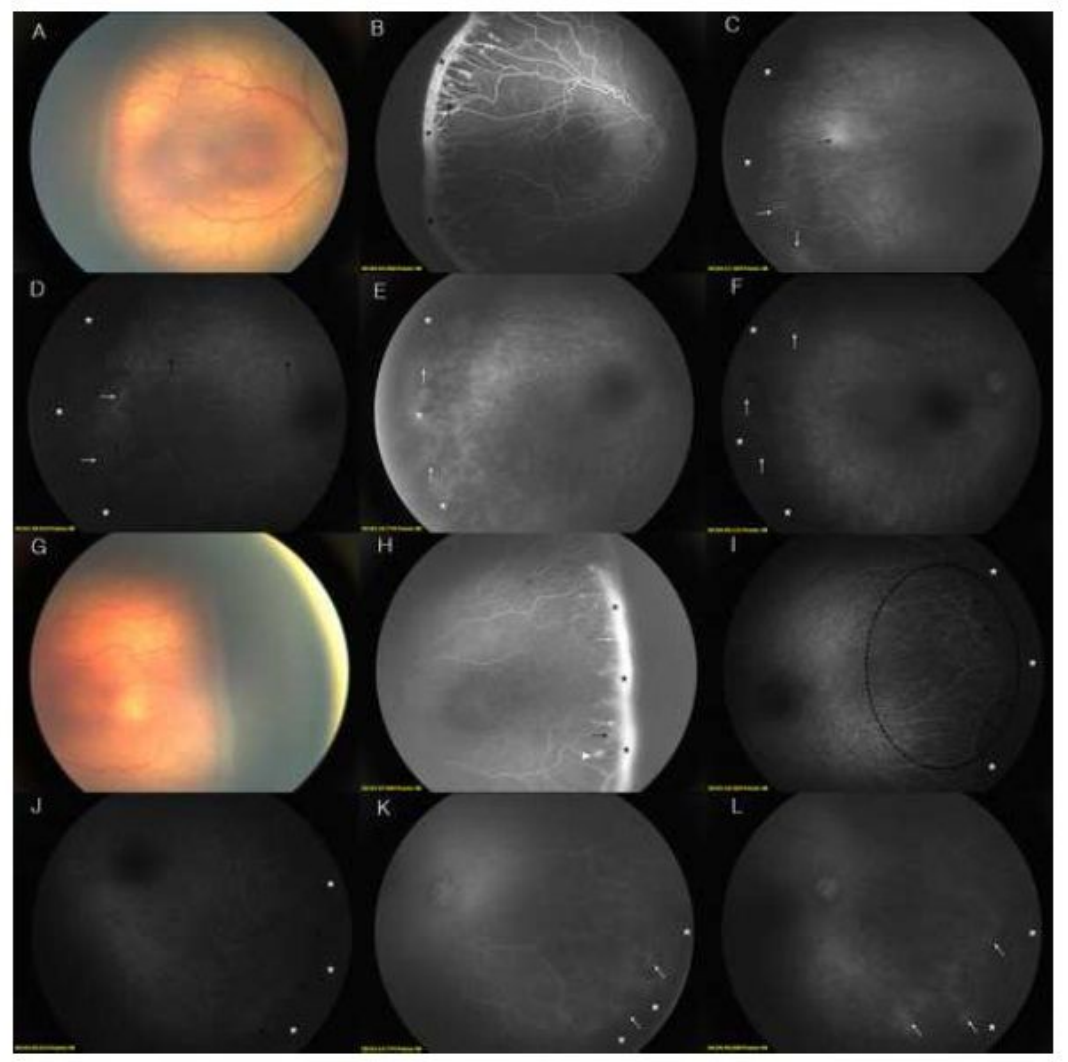

Figure 7

Fluorescein angiography of a premature baby after treated with bevacizumab, white arrows indicating rosary-bead-like hyperfluorescent lesions inside the large vessels at late venous phase. 


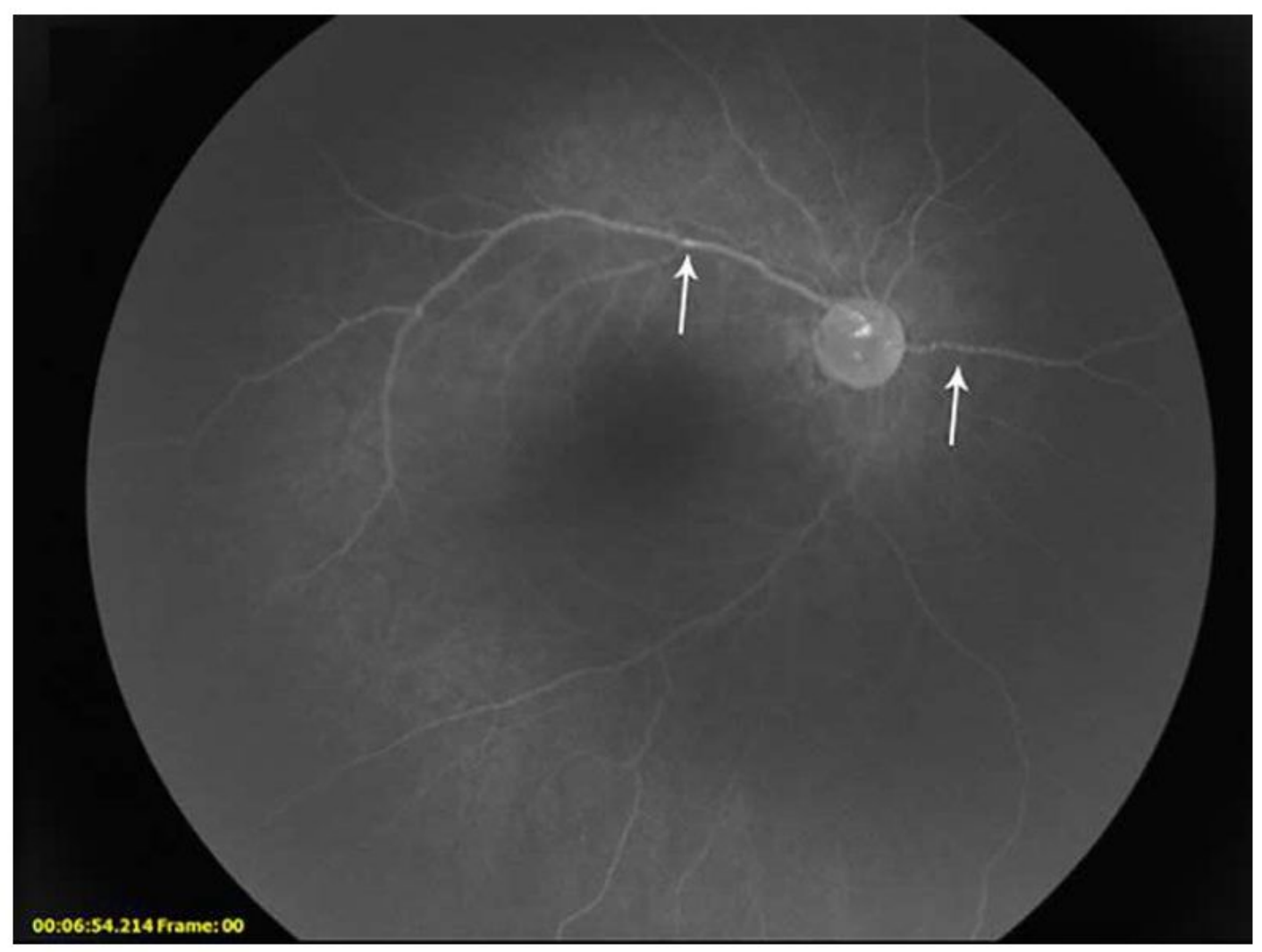

\section{Figure 8}

Fluorescein angiography images of a 4-year-old premature child treated with bevacizumab in both eyes (A-F), large avascular retinal areas beyond the $\mathrm{V}$-Av $\mathrm{J}$ (white asterisks), large tufts (black arrows), abnormal non-dichotomous branching pattern at the large, small and precapillary level of retinal vessels (white arrows), widespread shunt vessels and odd-shaped anastomoses and branches detected in both the vascularized retina and the V-Av $\mathrm{J}$ (black arrowheads), appearance of capillary network inside retinal vascularized zone (white arrowheads), areas of hypofluorescence due to the loss of periarteriolar capillary bed (black asterisks). vascular-avascular junction: V-Av J

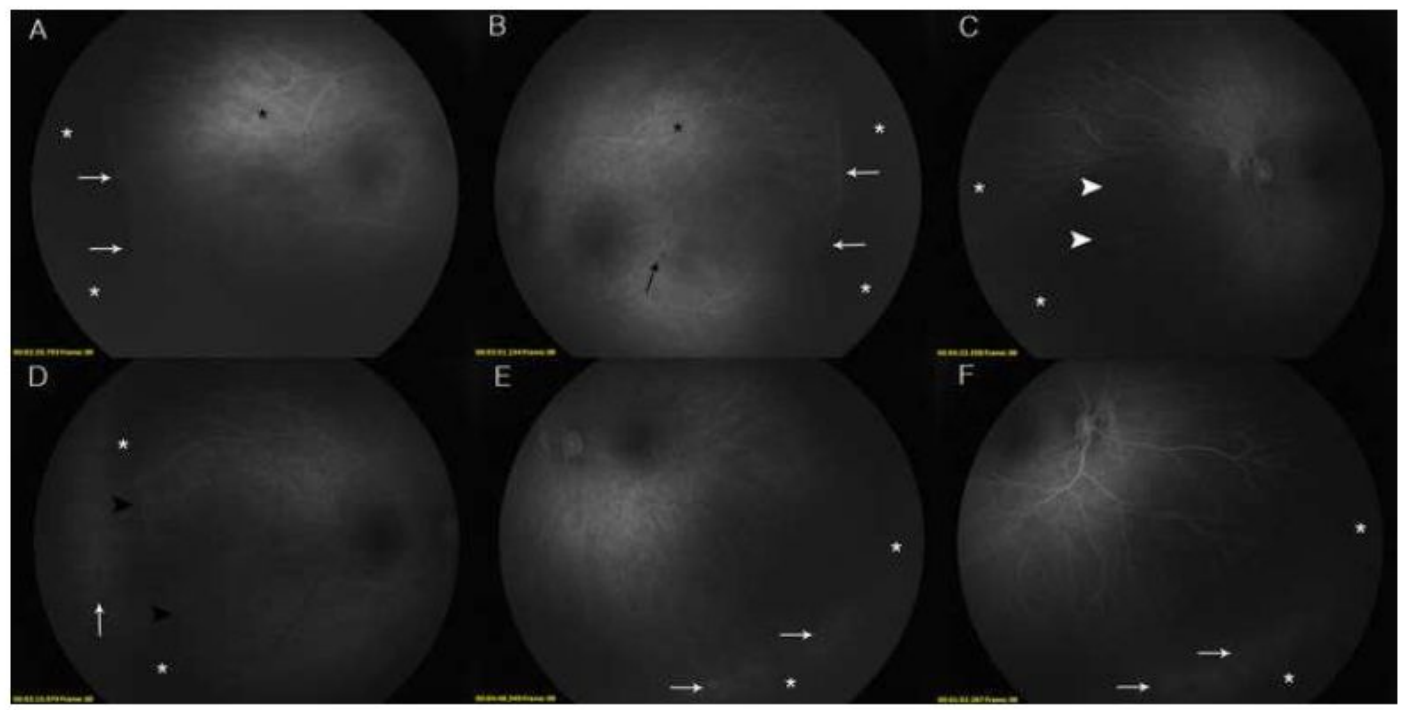

\section{Figure 9}

RetCam color fundus photographs (A-F) and fluorescein angiography images (G-I) belonging to a 5-year-old patient whose vascularization was completed. The images represent respectively: (A, B, D, and E), before treatment; (C and F), 15 months after treatment, (G-I) 27 months after treatment. C, F, G-I, lasting tortuosity of the retinal vessels, $\mathrm{H}$, abnormal development of the retinal vasculature with the formation of shunts between large vessels in the posterior pole, and narrowing of the FAZ (black dotted circle), and tiny vascular loops (black arrows), corkscrew-like anastomose (white arrow), hypofluorescent areas within the vascularized retina among hyperfluorescent areas (white dotted circle), G-I, completed peripheral retinal vascularization. F and I, 
peripapillary atrophy at the temporal side of the optic disc (black arrowhead), narrowing of the FAZ (black dotted circle), soft oblique-angled vessel extending from large to small level, developing shunts between vessels and ending with besom-like branching at small level (white arrowheads). foveal avascular zone: FAZ

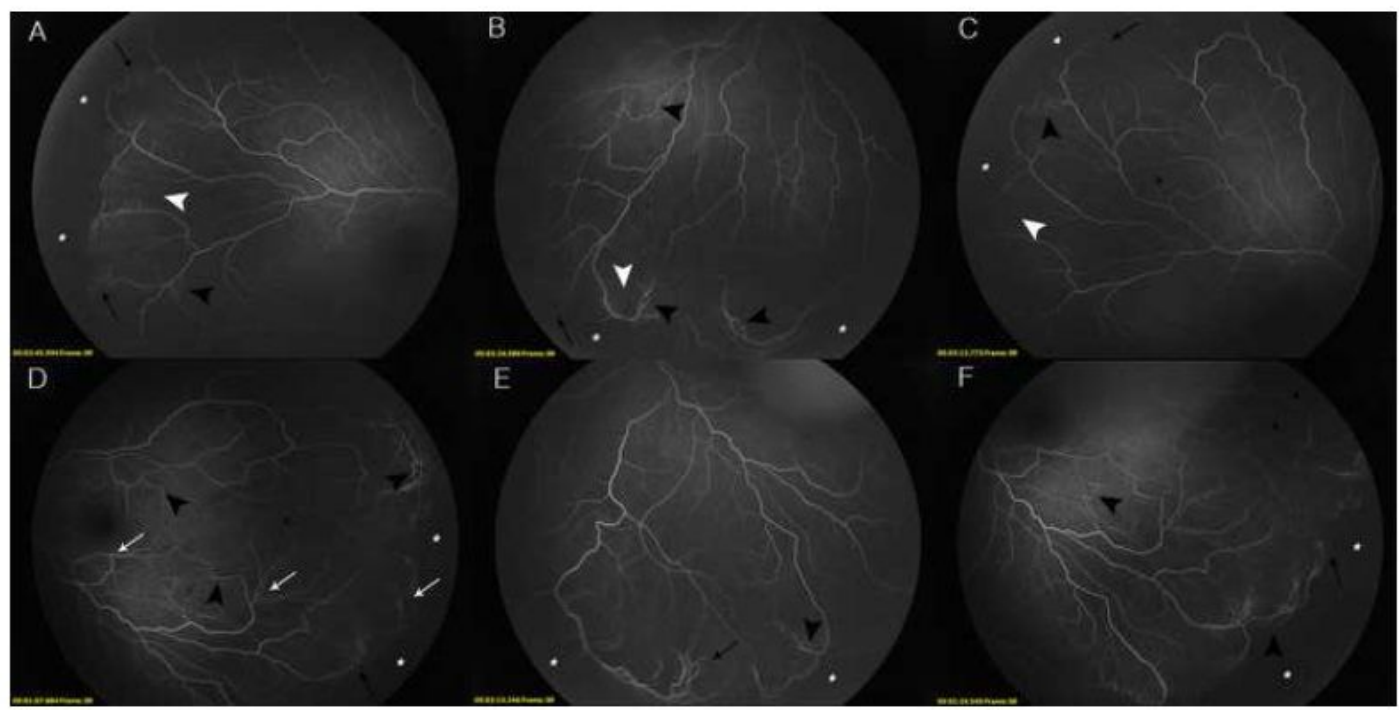

\section{Figure 10}

Fluorescein angiography images displaying the features of the retina of a 6-year-old. A-D). A-D, large tuft formation and capillary tufts with fluorescein leakage (black asterisk), A, tuft-shaped immature and incomplete vascularization (black arrows) in avascular peripheral retina (white asterisks), irregular branching of large and middle size arterioles and chaotic anastomosis in vascularized retina (black circle), B, persistent tortuosity of the vessels (black arrowhead), large dark area (white dotted circle) including small darker fields (white arrowheads) due to capillary obliteration in the vascularised retina, with adjacent hyperfluorescence in the posterior pole (black percent sign), narrowing of the foveal avascular zone especially at superior quadrant of the fovea (black dotted circle), peripapillary temporal cresent atrophy (white arrow). C and D, due to the leaking from tuft-shaped immature vessels, retinal mottling at late phase of the angiography (white circle).

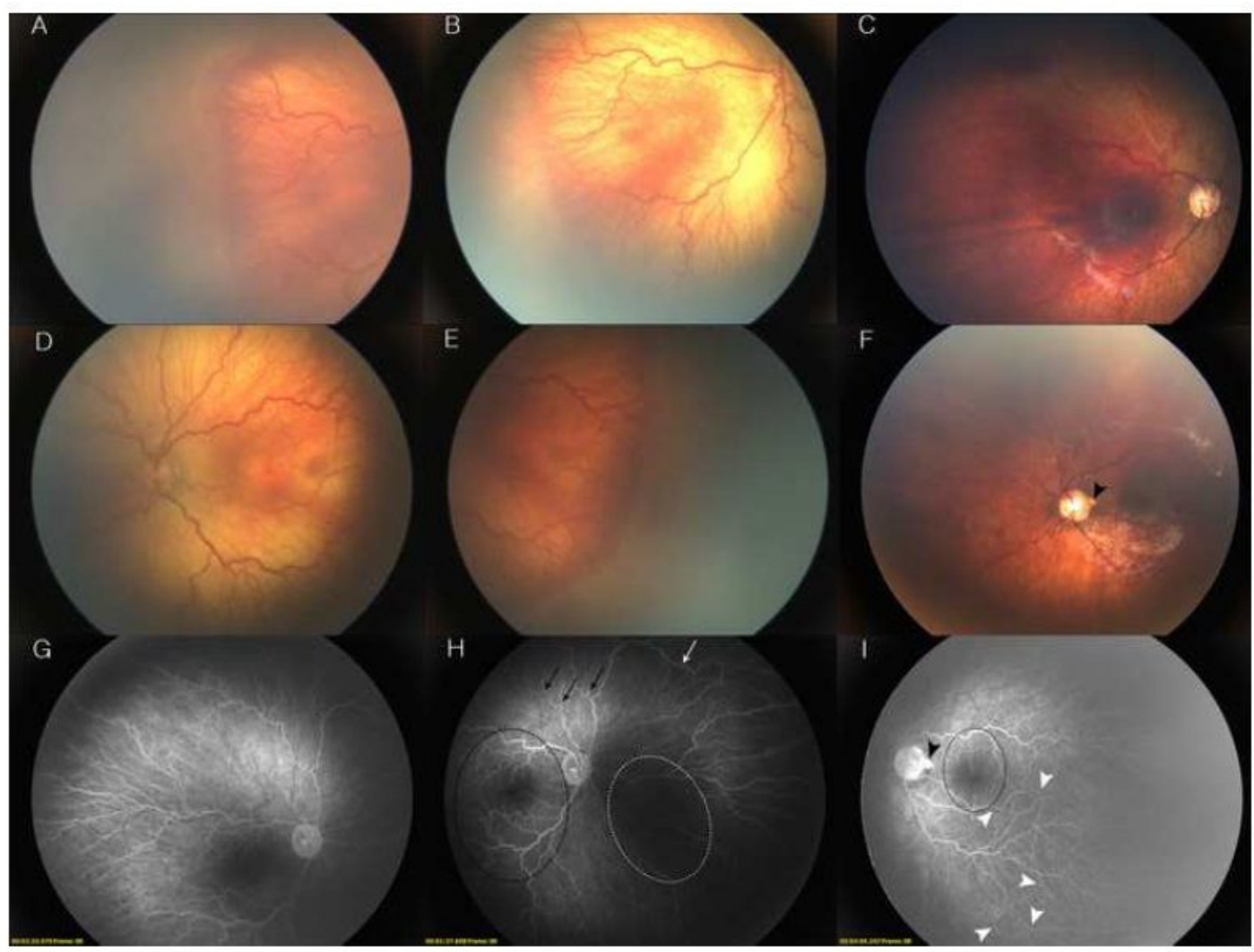

Figure 11 
Fluorescein angiography (FA) images taken at age of 2 years (A-C) and at age of 6 years (D-F). A and D, permanent circumferential vessel formation (black arrows) with wide arteriovenous anastomosis in vascularized retina. A-F, immature, fuzzy capillary network (white arrows) between the medium and small vessels and extending to the retinal periphery. A-C, significant leakage from small vessels and capillaries (white circle). D-F, vascular leaks still in progress although severity decreases (white circle). A-F, hyperfluorescent dotted lesions (white arrowhead) and hypofluorescence (black arrowhead) in retinal vascularised area. B, extensive hyperfluorescence and hypofluorescent area due to capillary dropout among vessels (black and white percent signs, respectively). A-F, large areas of avascular retina (white asterisk).

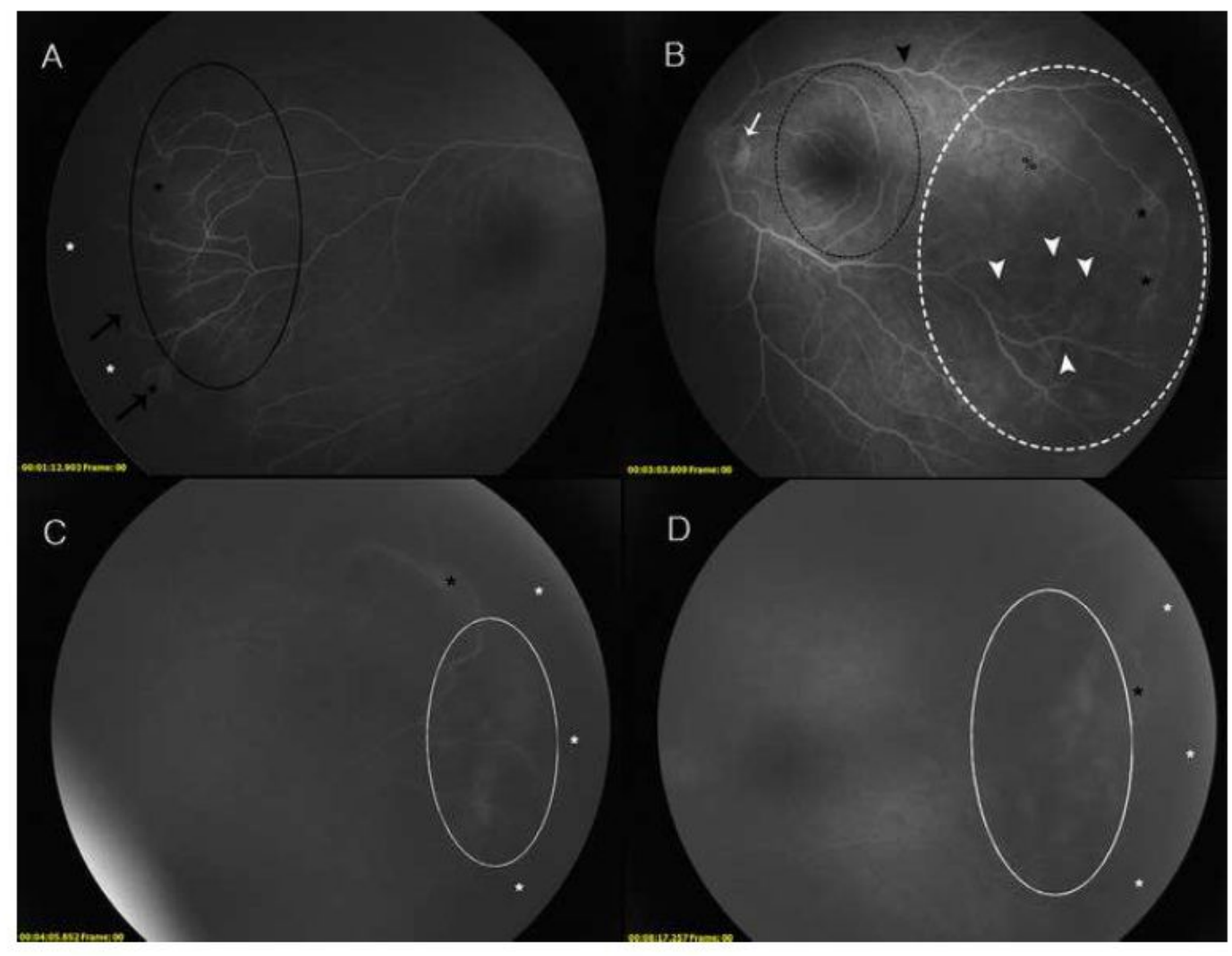

\section{Figure 12}

Fluorescein angiography images showing choroidal filling patterns in different cases. In all cases, dark area of retinal capillary obliteration is observed in the vascularized retina. A, one month old, lobular, B, 3 years old, linear, C, 2 years old, linear-lobular, D, 6 months old, lobular choroidal filling pattern with large hypofluorescent areas related to diffuse abnormal choriocapillary perfusion (white asterisks). 